\title{
Selection of Species Specific Panel of Reference Genes Across Native Livestock Species Adapted to Trans-himalayan Region of Leh-ladakh
}

\author{
Manish Tiwari \\ National Bureau of Animal Genetic Resources \\ Monika Sodhi \\ National Bureau of Animal Genetic Resources \\ Preeti Verma \\ National Bureau of Animal Genetic Resources \\ Prince Vivek \\ National Bureau of Animal Genetic Resources \\ Ranjit Kataria \\ National Bureau of Animal Genetic Resources \\ Saket Niranjan \\ National Bureau of Animal Genetic Resources \\ Vijay Bharti \\ DRD O-Defense Institute of High-altitude Research, Leh, India

\section{Nampher Masharing} \\ National Bureau of Animal Genetic Resources \\ Gayatri Gujar \\ National Bureau of Animal Genetic Resources

\section{Divya Chanda} \\ National Bureau of Animal Genetic Resources \\ Manishi Mukesh ( $\square$ mmukesh_26@hotmail.com) \\ National Bureau of Animal Genetic Resources
}

\section{Research Article}

Keywords: species specific panel, genes, native livestock species, trans-Himalayan region, Leh-Ladakh

Posted Date: January 19th, 2022

DOI: https://doi.org/10.21203/rs.3.rs-727900/v2

License: (c) (i) This work is licensed under a Creative Commons Attribution 4.0 International License. Read Full License 


\section{Abstract}

The identification of appropriate references genes is an integral component of any gene expression-based study for getting accuracy and reliability in data interpretation. In this study, we evaluated the expression stability of 10 candidate reference genes (GAPDH, RPL4, EEF1A1, RPS9, HPRT1, UXT, RPS23, B2M, RPS15, ACTB) in peripheral blood mononuclear cells of livestock species that are adapted to high altitude hypoxia conditions of Leh-Ladakh. A total of 37 PBMCs samples from six native livestock species of leh-Ladakh region such as Ladakhi cattle (LAC), Ladakhi yak (LAY), Ladakhi donkey (LAD), Chanthangi goat (CHG), Double hump cattle (DHC) and Zanskar ponies (ZAP) were included in this study. The commonly used statistical algorithms such as geNorm, NormFinder, BestKeeper and RefFinder were employed to assess the stability of these RGs in all the livestock species. Our study has identified different panel of reference genes in each species; for example, EEF1A1, RPL4 in Ladakhi cattle; GAPDH, RPS9, ACTB in Ladakhi yak; HPRT1, B2M, ACTB in Ladakhi donkey; HPRT1, B2M, $A C T B$ in Double hump camel, RPS9, HPRT1 in Changthangi goat, HPRT1 and ACTB in Zanskar ponies. To the best of our knowledge, this is the first systematic attempt to identify panel of RGs across different livestock species types adapted to high altitude hypoxia conditions. In future, the findings of the present study would be quite helpful in conducting any transcriptional studies to understand the molecular basis of high altitude adaptation of native livestock population of LehLadakh.

\section{Introduction}

In recent years, high-throughput techniques such as serial analysis of gene expression (SAGE), expressed sequence tag (EST), microarray and RNA-seq have been widely employed to study the gene functions and understand the transcriptional regulations in humans, animals as well as plants ${ }^{1-5}$. However, the high throughput expression data requires validation using real-time quantitative polymerase chain reaction (qPCR). The qPCR technique because of its dynamic range, scalability, sensitivity, and reproducibility has always been considered as precise technique to estimate the relative abundance of mRNA transcripts in any cell types ${ }^{6-9}$. However, in order to perform appropriate gene expression analysis, it has become mandatory to select stable reference genes (RGs) that can normalize provide accurate and reliable qPCR results for each and every experimental condition ${ }^{10}$. This has become the most popular approach to normalize the qPCR-based gene expression data as evident from numerous publications across mouse ${ }^{11-13}$, human ${ }^{5,14}$ plants $^{15,16}$ and livestock species ${ }^{17-21}$. Lack of appropriate RGs can greatly compromise the reliability of qPCR due to technical variations or errors arises during sample preparation, like quality and starting amount of RNA, efficiency of reverse transcription, efficiency of PCR and errors during pipetting $^{22}$. All these technical variations will affect both the target genes as well as selected panel of RGs. Therefore, it's important to normalize the gene expression data by identifying suitable RGs or internal control genes (ICGs) in order to obtain an accurate and reliable gene expression data. Identification and validation of appropriate RGs has thus become an essential component in any gene expression studies wherein RGs are exposed to the same experimental conditions as target genes ${ }^{23}$.

Ladakh, the newly formed union territory in the northern most region of India bordering China and Pakistan is one of the world's highest inhabited region (3,500-5,500 m above sea level) surrounded by snow-capped Himalayan, Zanskar and Karakoram ranges. The cold-arid desert of Ladakh is characterized by harsh climatic conditions such as extreme temperature variations, ranging from $-40^{\circ} \mathrm{C}$ in winter and $35^{\circ} \mathrm{C}$ in summer; low humidity (25-40\%), low precipitation (80-300 mm) and low oxygen level (nearly $60-70 \%$ of the oxygen concentration at sea level); high UV radiations and wind erosion.

In such a difficult terrain of Ladakh, where land resources are meager, animal wealth plays an important role in the life of the local people. Ladakh is blessed with several unique native animal genetic resources such as yak, cattle, dzomo, dzo, goat, sheep, donkeys, horses, and double hump camel. Each of these species living has a unique ability to adapt themselves to chronic hypoxia and low ambient temperature. The economy of local people is mainly dependent on these livestock species. The native cattle known as "Ladakhi cattle" (Bos indicus) is a unique germplasm having excellent adaptation potential to high altitude hypobaric stress. In spite of extreme climatic conditions, subsistence on poor quality feed and low availability of water, it provides around $2.5-4.5 \mathrm{~kg}$ of milk and thus serves as an important source of animal protein for the local people, 
especially during lean winter period. Similar to Ladakhi cattle, local yak population (Bos grunniens) are also the major resources of milk and milk products for the local people. The locally made butter and churpi from yak and local cow are always in high demand in the local market. The Ladakhi goat (Capra hircus) commonly known as Changthangi goat, or world famous pashmina goat is mainly reared for meat, milk \& fiber (Pashmina and Mohair), hide and skin. Ladakhi donkey (Equus asinus) and Zanskari ponies (Equus caballus) is yet another important animal genetic resources in the region that serves as an important pack animal for the local people and Indian army. The Zanskari ponies are medium size mountain horse and very well adapted to work at higher altitudes. Another unique species; double hump camel (Camelus bactrianus) is quite popular amongst tourists especially for safari in world famous cold desert stretch of Nubra valley region of Ladakh. In last five years, our group at ICAR-NBAGR, Karnal in collaboration with DRDO-DIHAR, Leh and Animal Husbandry Department, Leh has initiated the efforts to characterize the livestock breeds of Ladakh. Under this programme, the native cattle, yak and donkey populations of Ladakh have been studied for both phenotypic and genotypic characterization. We have also made efforts to identify genes and pathways responsible for high altitude adaptation in Ladakhi cattle ${ }^{24,25}$. Each of these species has developed effective mechanism to survive at high altitude and low oxygen condition. Under such adverse climatic conditions, the survival and performance of exotic breeds is not a viable option in hypoxia condition. It only allows the well adapted animal genetic resources to thrive and perform. Therefore, understanding transcriptome signatures and identifying genes highly abundant across all these species will provide strong clue on molecular mechanism operating at transcriptional level in response to abiotic hypoxia stress across these species. By making such advancements, not only these resources will be characterized and documented but will also help to understand these unique animals production attribute in a better way for future exploitation and overall improvement. As a step forward, the present study was designed to identify and select panel of stably expressed RGs for future transcriptional studies in each of the six livestock species of Ladakh.

In recent past, numerous studies have been conducted in similar lines to identify panel of appropriate RGs in several livestock

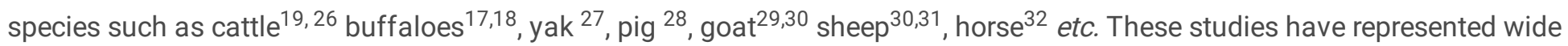
array of environmental or experimental conditions such as responses to external stimuli (heat stress, endurance, exercise), physiological or developmental stages, lactation cycle, cellular response, etc. ${ }^{25,19,17,18,27,33,34,32}$. It is now evident that set of RGs that perform well in one particular condition or species may not work well in other experimental conditions or other species. Therefore, in the present study, an effort was made to evaluate and identify panel of appropriate RGs in peripheral blood mononuclear cells (PBMCs) of six livestock species well adapted to high altitude region of Leh-Ladakh viz., Ladakhi cattle, Ladakhi yak, Ladakhi donkey, Changthangi goat, Zanskar ponies and double hump camel. All these livestock species are native of Leh and Ladakh and have been naturally selected not only to sustain but perform and reproduce well under high altitude hypoxia stressful conditions. The 10 candidate RGs that were evaluated in the present study were; glyceraldehyde 3phosphate dehydrogenase (GAPDH), beta-Actin $(A C T B)$, ubiquitously expressed transcript (UXT), ribosomal protein S15A (RPS15A), beta 2-microglobulin (B2M), ribosomal protein L-4 (RPL4), ribosomal proteinS18 (RPS18), ribosomal protein S9 (RPS9), ribosomal protein S23 (RPS23), hydroxymethylbilane synthase (HMBS), hypoxanthine phosphoribosyl transferase (HPRT1).

\section{Results}

\section{Specificity, expression abundance and coefficient of variation of individual RGs}

In the present study, an effort was made to identify the appropriate RGs in all the major livestock species that are native of Leh-ladakh region viz., Ladakhi cattle (LAC), Ladakhi yak (LAY), Ladakhi donkey (LAD), Double hump camel (DHC), Changthangi goat ( $\mathrm{CHG}$ ), Zanskar ponies (ZAP). The specificity of each primer pair was confirmed by the specific amplification checked in agarose gel and presence of single peak in melt curve analysis. The correlation coefficient $\left(R^{2}\right)$ and amplification efficiency (E) for individual primer pair in each of the six livestock species are given in Table 1. The expression abundance of individual RGs in each species is shown in Box Whisker plot (Fig. 1a-f). The Ct values of individual RGs ranged from RPS23 (13.94) to HPRT1 (30.18) in LAC; RPS23 (14.37) to RPS15 (33.82) in LAY; RPS23 (13.86) to RPS15 (35.47) in LAD; 
RPS15 (16.05) to RPS23 (34.90) in DHC; RPS15 (13.63) to RPS23 (34.92) in CHG; RPS15 (16.06) to RPS23 (36.06) in ZAP (Table 2).

Table 1

Gene symbol, primer sequence, melting temperature $\left(T_{a}\right)$, amplicon size, slope, PCR efficiency and $R^{2}$ of RGs for each evaluated RG

\begin{tabular}{|c|c|c|c|c|c|c|}
\hline Gene Symbol & Primers 5'-3' (Forward, Reverse) & $\mathrm{T}_{\mathrm{a}}(\mathrm{C})$ & $\begin{array}{l}\text { Amplicon } \\
\text { Size (bp) }\end{array}$ & Slope & $\begin{array}{l}\text { PCR } \\
\text { efficiency }\end{array}$ & $\mathrm{R}^{2}$ \\
\hline \multirow[t]{2}{*}{ Beta-Actin $(A C T B)$} & F:5'GCGTGGCTACAGCTTCACC3' & \multirow[t]{2}{*}{60} & \multirow[t]{2}{*}{56} & \multirow[t]{2}{*}{-3.10} & \multirow[t]{2}{*}{107.40} & \multirow[t]{2}{*}{0.997} \\
\hline & R:3'TTGATGTCACGGACGATTTC5' & & & & & \\
\hline \multirow{2}{*}{$\begin{array}{l}\text { Glyceraldehyde 3-phosphate } \\
\text { dehydrogenase (GAPDH) }\end{array}$} & F:5'TGGAAAGGCCATCACCATT3' & \multirow[t]{2}{*}{60} & \multirow[t]{2}{*}{60} & \multirow[t]{2}{*}{-2.99} & \multirow[t]{2}{*}{119.28} & \multirow[t]{2}{*}{0.997} \\
\hline & R:3'CCCACTTGATGTTGGCAG5' & & & & & \\
\hline \multirow{2}{*}{$\begin{array}{l}\text { Eukaryotic translation } \\
\text { elongation factor } 1 \text { alpha } \\
1(E E F 1 A 1)\end{array}$} & F:5'CATCCCAGGCTGACTGTGC3' & \multirow[t]{2}{*}{60} & \multirow[t]{2}{*}{101} & \multirow[t]{2}{*}{-3.11} & \multirow[t]{2}{*}{109.65} & \multirow[t]{2}{*}{0.998} \\
\hline & R:3'TGTAAGCCAAAAGGGCATG5' & & & & & \\
\hline \multirow[t]{2}{*}{$\beta 2$ Microglobulin (B2M) } & F:5'CTGCTATGTGTATGGGTTCC3' & \multirow[t]{2}{*}{60} & \multirow[t]{2}{*}{101} & \multirow[t]{2}{*}{-3.03} & \multirow[t]{2}{*}{114.64} & \multirow[t]{2}{*}{0.999} \\
\hline & R:3'GGAGTGAACTCAGCGTG5' & & & & & \\
\hline \multirow[t]{2}{*}{ Ribosomal protein L4 (RPL4) } & F:5'TTGGAAACATGTGTCGTGG3' & \multirow[t]{2}{*}{60} & \multirow[t]{2}{*}{101} & \multirow[t]{2}{*}{-3.12} & \multirow[t]{2}{*}{109.45} & \multirow[t]{2}{*}{0.998} \\
\hline & R:3'GCAGATGGCGTATCGCTTCT5' & & & & & \\
\hline \multirow{2}{*}{$\begin{array}{l}\text { Ribosomal protein } \mathrm{S} 15 \\
\text { (RPS15) }\end{array}$} & F:5'GAATGGTGCGCATGAATGT3' & \multirow[t]{2}{*}{60} & \multirow[t]{2}{*}{101} & \multirow[t]{2}{*}{-2.89} & \multirow[t]{2}{*}{127.12} & 0.996 \\
\hline & R:3'GACTTTGGAGCACGGCCTA5' & & & & & \\
\hline Ribosomal protein S15 & F:5'CCCAATGATGGTTGCTTGAA3' & 60 & 101 & -3.20 & 102.27 & 0.990 \\
\hline & R:3'CGGACTCCAGGAATGTCAC5' & & & & & \\
\hline Ribosomal protein S9 (RPS9) & F:5'CCTCGACCAAGAGCTGAAG3' & 60 & 54 & -3.03 & 113.54 & 0.996 \\
\hline & R:3'CCTCCAGACCTCACGTTTGT5' & & & & & \\
\hline Ubiquitously & F:5'TGTGGCCCTTGGATATGGTT3' & 60 & 101 & -3.33 & 99.36 & 0.988 \\
\hline expressed transcript $(U X T)$ & R:3'GGTTGTCGCTGAGCTCTGTG5' & & & & & \\
\hline Hypoxanthine Phosphoribosyl & F:5'GAGAAGTCCGAGTTGAGTT3' & 60 & 101 & -3.03 & 113.60 & 0.988 \\
\hline & R:3'GGCTCGTAGTGCAAATGAA5' & & & & & \\
\hline
\end{tabular}


Table 2

The average raw $\mathrm{Ct}$ values of individual $\mathrm{RG}$ s in different species

\begin{tabular}{|c|c|c|c|c|c|c|c|c|c|c|c|}
\hline S.No & SAMPLE & GAPDH & ACTB & RPS9 & EEF1A1 & RPS15 & RPS23 & $U X T$ & RPL4 & $B 2 M$ & HPRT1 \\
\hline 1 & LAC 1 & 22.166 & 24.350 & 22.395 & 18.810 & 19.511 & 15.934 & 26.065 & 21.560 & 19.355 & 30.175 \\
\hline 2 & LAC 2 & 21.865 & 22.880 & 20.150 & 17.320 & 19.785 & 14.275 & 23.860 & 20.055 & 18.195 & 28.375 \\
\hline 3 & LAC 3 & 22.080 & 23.515 & 19.975 & 16.895 & 19.550 & 14.240 & 24.175 & 19.665 & 18.715 & 27.950 \\
\hline 4 & LAC 4 & 22.295 & 24.660 & 19.865 & 17.260 & 19.570 & 14.094 & 24.545 & 20.145 & 18.565 & 28.135 \\
\hline 5 & LAC 5 & 22.525 & 22.640 & 20.035 & 17.090 & 19.205 & 13.943 & 24.675 & 19.605 & 18.745 & 28.865 \\
\hline 6 & LAC 6 & 22.065 & 24.900 & 20.410 & 17.225 & 19.445 & 14.062 & 24.505 & 19.750 & 17.735 & 29.690 \\
\hline 7 & LAY 11 & 18.984 & 22.124 & 22.675 & 23.616 & 33.537 & 17.509 & 22.220 & 23.867 & 22.913 & 24.790 \\
\hline 8 & LAY 12 & 17.341 & 20.518 & 21.299 & 20.124 & 29.057 & 15.891 & 22.872 & 24.407 & 20.860 & 24.951 \\
\hline 9 & LAY 15 & 17.458 & 19.617 & 21.063 & 19.573 & 30.821 & 14.365 & 22.848 & 25.646 & 18.109 & 24.333 \\
\hline 10 & LAY 16 & 18.306 & 21.148 & 22.322 & 23.314 & 30.667 & 15.700 & 23.041 & 23.709 & 22.436 & 25.037 \\
\hline 11 & LAY 18 & 19.202 & 21.497 & 23.420 & 23.584 & 33.815 & 16.677 & 22.838 & 24.405 & 22.954 & 25.410 \\
\hline 12 & LAY 19 & 18.548 & 20.981 & 22.407 & 23.161 & 33.664 & 16.822 & 22.763 & 23.866 & 21.809 & 25.026 \\
\hline 13 & LAD 1 & 22.631 & 21.085 & 19.703 & 18.913 & 34.072 & 14.334 & 23.918 & 21.676 & 17.632 & 25.613 \\
\hline 14 & LAD 2 & 24.527 & 20.870 & 20.745 & 20.654 & 31.857 & 14.842 & 22.433 & 21.309 & 18.578 & 26.040 \\
\hline 15 & LAD 3 & 20.735 & 20.623 & 19.752 & 17.940 & 33.395 & 14.630 & 23.017 & 23.167 & 16.685 & 24.795 \\
\hline 16 & LAD 4 & 23.579 & 19.967 & 22.779 & 19.457 & 34.096 & 14.027 & 22.706 & 21.676 & 18.105 & 25.826 \\
\hline 17 & LAD 5 & 22.868 & 20.767 & 24.162 & 20.442 & 35.473 & 13.857 & 21.455 & 20.553 & 17.750 & 25.568 \\
\hline 18 & CHG 27 & 14.350 & 18.640 & 19.792 & 18.836 & 34.923 & 13.633 & 21.406 & 23.870 & 28.316 & 25.075 \\
\hline 19 & CHG 28 & 15.877 & 18.359 & 19.575 & 20.644 & 30.897 & 15.230 & 20.443 & 20.810 & 33.046 & 25.448 \\
\hline 20 & CHG 29 & 17.404 & 17.562 & 19.358 & 18.338 & 34.623 & 15.027 & 22.621 & 23.095 & 32.082 & 24.355 \\
\hline 21 & CHG 30 & 16.321 & 18.014 & 19.434 & 20.044 & 33.763 & 14.099 & 20.786 & 20.015 & 31.783 & 24.553 \\
\hline 22 & CHG 33 & 15.238 & 17.090 & 19.151 & 17.746 & 30.183 & 13.943 & 21.771 & 22.071 & 26.700 & 24.858 \\
\hline 23 & DHC 34 & 23.103 & 18.367 & 19.258 & 17.584 & 30.718 & 17.048 & 18.195 & 18.228 & 20.174 & 21.929 \\
\hline 24 & DHC 35 & 22.565 & 19.002 & 19.688 & 17.167 & 33.965 & 16.055 & 20.889 & 22.323 & 21.401 & 22.778 \\
\hline 25 & DHC 37 & 20.412 & 19.155 & 18.550 & 17.387 & 31.888 & 16.886 & 19.762 & 20.840 & 19.244 & 22.105 \\
\hline 26 & DHC 38 & 24.128 & 19.254 & 19.583 & 17.930 & 34.898 & 17.266 & 19.434 & 18.681 & 20.421 & 22.271 \\
\hline 27 & DHC 39 & 22.619 & 19.997 & 20.835 & 16.869 & 30.624 & 17.983 & 20.106 & 21.816 & 20.867 & 22.271 \\
\hline 28 & ZAP 1 & 23.198 & 16.494 & 21.355 & 20.253 & 34.904 & 18.130 & 27.172 & 19.203 & 16.340 & 22.058 \\
\hline 29 & ZAP 2 & 19.785 & 16.688 & 21.265 & 19.610 & 36.059 & 17.718 & 27.222 & 18.761 & 16.059 & 25.723 \\
\hline 30 & ZAP 3 & 20.695 & 18.157 & 21.454 & 20.502 & 36.064 & 17.868 & 27.144 & 19.211 & 20.486 & 26.809 \\
\hline 31 & ZAP 4 & 25.664 & 19.940 & 19.839 & 18.813 & 33.605 & 16.471 & 26.166 & 17.547 & 19.799 & 24.798 \\
\hline 32 & ZAP 5 & 19.687 & 16.401 & 21.097 & 19.991 & 34.567 & 17.589 & 27.407 & 18.887 & 16.074 & 25.422 \\
\hline
\end{tabular}




\section{Expression stability analysis of RGs in each livestock species}

\section{Ladakhi Cattle (LAC)}

The geNorm analysis ranked candidate reference genes as per their mean expression stability value (M value) which was below the threshold value of 1.5 for all the $10 \mathrm{RGs}$. The ranking order based on $\mathrm{M}$ value were $E E F 1 A 1=R P L 4>R P S 23>R P S 9>$ $U X T>B 2 M>G A P D H>R P S 15>H P R T 1>A C T B$ (Fig. 2a). The M value ranged from 0.147 (EEF1A1) to 0.689 (ACTB). The lower $M$ value indicates higher expression stability while higher $\mathrm{M}$ value indicates lower expression stability. On the basis of $\mathrm{M}$ value, $E E F 1 A 1=R P L 4 \mathrm{RG}$ pair was most stable expressed while $A C T B$ was least stable. Another parameter that was evaluated by geNorm was the pairwise variation $\mathrm{Vn} / \mathrm{n}+1$ in order to calculate the optimal number of RGs to be required for normalization. The pairwise variation (V) score of all the RGs were below 0.15 (Fig. 2b) which is an ideal pairwise recommended score ${ }^{35}$. Therefore, as per $\mathrm{V}$ value, combination of two RGs could be suggested to normalize the qPCR data in PBMCs of Ladakhi cattle.

In NormFinder analysis as well, the ranking stability of individual RGs were decided by the lower values indicating higher stability. In LAC, Normfinder analysis resulted in same panel of stable RGs (EEF1A1, RPL4, UXT, RPS23,) as identified in geNorm analysis. On the other hand, ACTB, HPRT1, RPS15 RGs were identified as least stable. The ranking order from most to least stable RGs was as follows: EEF1A1>RPL4>UXT>RPS23>RPS9>B2M>GAPDH>RPS15> HPRT1>ACT (Fig. 2c \& Table 3). 
Table 3

Overall Ranking of Best suitable RGs across different species

\begin{tabular}{|c|c|c|c|c|c|c|}
\hline Species & Ranking & geNorm & Normfinder & Bestkeeper & & RefFinder \\
\hline & & M value & Stability value & STDEV & coff. of corr & \\
\hline \multirow[t]{10}{*}{$\begin{array}{l}\text { Ladakhi cattle } \\
\text { (LAC) }\end{array}$} & 1 & EEF1A1(0.147) & $\begin{array}{l}\text { EEF1A1 } \\
(0.171)\end{array}$ & RPS15 (0.123) & $\begin{array}{l}\text { EEF1A1 } \\
(0.978)\end{array}$ & $\begin{array}{l}\text { EEF1A1 } \\
(1.41)\end{array}$ \\
\hline & 2 & RPL4(0.147) & RPL4 (0.278) & $\begin{array}{l}\text { GAPDH } \\
(0.163)\end{array}$ & RPS9 (0.973) & RPL4 (2.11) \\
\hline & 3 & RPS23 (0.186) & UXT (0.297) & B2M (0.391) & RPS23 (0.973) & RPS23 (3.98) \\
\hline & 4 & RPS9 (0.267) & RPS23 (0.301) & $\begin{array}{l}\text { EEF1A1 } \\
(0.468)\end{array}$ & RPL4 (0.959) & UXT (4.36) \\
\hline & 5 & UXT (0.322) & RPS9 (0.498) & RPL4 (0.481) & UXT (0.941) & RPS15 (4.76) \\
\hline & 6 & B2M (0.413) & B2M (0.502) & UXT (0.489) & HPRT1 (0.763) & B2M (5.05) \\
\hline & 7 & $\begin{array}{l}\text { GAPDH } \\
(0.512)\end{array}$ & $\begin{array}{l}\text { GAPDH } \\
(0.597)\end{array}$ & RPS23 (0.503) & B2M (0.637) & GAPDH (5.12) \\
\hline & 8 & RPS15 (0.563) & RPS15 (0.602) & RPS9 (0.642) & АСТВ (0.433) & RPS9 (5.32) \\
\hline & 9 & HPRT1 (0.605) & HPRT1 (0.616) & HPRT1 (0.712) & $\begin{array}{l}\text { GAPDH } \\
(0.017)\end{array}$ & HPRT1 (9) \\
\hline & 10 & ACTB (0.689) & АСТВ (0.922) & ACTB (0.812) & RPS15 (0.001) & ACTB (10) \\
\hline \multirow{10}{*}{$\begin{array}{l}\text { Ladakhi } \\
\text { Yak } \\
\text { (LAY) }\end{array}$} & 1 & $\begin{array}{l}\text { GAPDH } \\
(0.223)\end{array}$ & $\begin{array}{l}\text { GAPDH } \\
(0.112)\end{array}$ & UXT (0.180) & $\begin{array}{l}\text { GAPDH } \\
(0.967)\end{array}$ & GAPDH (1.41) \\
\hline & 2 & RPS9 (0.223) & RPS9 (0.112) & HPRT1 (0.240) & RPS9 (0.965) & RPS9 (2.21) \\
\hline & 3 & АСТВ (0.386) & ACTB (0.202) & RPL4 (0.500) & $\begin{array}{l}\text { EEF1A1 } \\
(0.960)\end{array}$ & АСTB (3.41) \\
\hline & 4 & RPS23 (0.507) & RPS23 (0.524) & $\begin{array}{l}\text { GAPDH } \\
(0.600)\end{array}$ & АСТВ (0.929) & HPRT1 (3.76) \\
\hline & 5 & HPRT1 (0.595) & HPRT1 (0.595) & ACTB (0.610) & B2M (0.927) & UXT (3.83) \\
\hline & 6 & UXT (0.708) & UXT (1.025) & RPS9 (0.680) & RPS23 (0.886) & RPS23 (4.86) \\
\hline & 7 & B2M (0.892) & $\begin{array}{l}\text { EEF1A1 } \\
(1.191)\end{array}$ & RPS23 (0.840) & RPS15 (0.813) & RPL4 (7.02) \\
\hline & 8 & $\begin{array}{l}\text { EEF1A1 } \\
(0.992)\end{array}$ & B2M (1.254) & B2M (1.350) & HPRT1 (0.727) & $\begin{array}{l}\text { EEF1A1 } \\
(7.71)\end{array}$ \\
\hline & 9 & RPL4 (1.121) & RPS15 (1.523) & $\begin{array}{l}\text { EEF1A1 } \\
(1.590)\end{array}$ & UXT (0.001) & B2M (7.74) \\
\hline & 10 & RPS15 (1.242) & RPL4 (1.538) & RPS15 (1.750) & RPL4 (0.001) & RPS15 (9.74) \\
\hline \multirow{5}{*}{$\begin{array}{l}\text { Ladakhi } \\
\text { Donkey } \\
\text { (LAD) }\end{array}$} & 1 & HPRT1(0.250) & HPRT1 (0.123) & ACTB (0.295) & HPRT1 (0.942) & HPRT1 (1.19) \\
\hline & 2 & B2M (0.250) & B2M (0.324) & HPRT1 (0.311) & $\begin{array}{l}\text { GAPDH } \\
(0.941)\end{array}$ & B2M (2.00) \\
\hline & 3 & RPS23 (0.571) & АСТВ $(0.518)$ & RPS23 (0.318) & B2M (0.940) & АСТВ (2.45) \\
\hline & 4 & АСTВ (0.612) & RPS23 (0.605) & B2M (0.472) & $\begin{array}{l}\text { EEF1A1 } \\
(0.927)\end{array}$ & RPS23 (3.46) \\
\hline & 5 & $\begin{array}{l}\text { EEF1A1 } \\
(0.751)\end{array}$ & $\begin{array}{l}\text { EEF1A1 } \\
(0.851)\end{array}$ & RPL4 (0.598) & RPS9 (0.619) & $\begin{array}{l}\text { EEF1A1 } \\
(5.44)\end{array}$ \\
\hline
\end{tabular}




\begin{tabular}{|c|c|c|c|c|c|c|}
\hline Species & Ranking & geNorm & Normfinder & Bestkeeper & & RefFinder \\
\hline & 6 & $\begin{array}{l}\text { GAPDH } \\
(0.857)\end{array}$ & UXT (1.181) & UXT (0.613) & RPS15 (0.022) & UXT (6.24) \\
\hline & 7 & UXT (0.984) & $\begin{array}{l}\text { GAPDH } \\
(1.208)\end{array}$ & $\begin{array}{l}\text { EEF1A1 } \\
(0.852)\end{array}$ & ACTB (0.001) & RPL4 (7.11) \\
\hline & 8 & RPL4 (1.090) & RPL4 (1.366) & RPS15 (0.92) & RPS23 (0.001) & GAPDH (7.17) \\
\hline & 9 & RPS15 (1.233) & RPS15 (1.377) & GAPDH (0.95) & UXT (0.00) & RPS15 (8.74) \\
\hline & 10 & RPSP (1.405) & RPS9 (1.912) & RPS9 (1.635) & RPL4 (0.001) & RPS9 (10.00) \\
\hline \multirow{10}{*}{$\begin{array}{l}\text { Chanthangi } \\
\text { Goat } \\
(\mathrm{CHG})\end{array}$} & 1 & RPS9(0.378) & RPS9 (0.310) & RPS9 (0.1760) & B2M (0.847) & RPS9 (1) \\
\hline & 2 & HPRT1 (0.378) & RPS23 (0.477) & HPRT1 (0.324) & RPS23 (0.676) & HPRT1 (2.38) \\
\hline & 3 & ACTB (0.434) & ACTB (0.486) & ACTB $(0.486)$ & $\begin{array}{l}\text { GAPDH } \\
(0.623)\end{array}$ & ACTB (2.71) \\
\hline & 4 & RPS23 (0.636) & HPRT1 (0.740) & RPS23 (0.595) & RPS15 (0.580) & RPS23 (3.13) \\
\hline & 5 & $\begin{array}{l}\text { EEF1A1 } \\
(0.803)\end{array}$ & $\begin{array}{l}\text { GAPDH } \\
(0.953)\end{array}$ & UXT (0.633) & $\begin{array}{l}\text { EEF1A1 } \\
(0.435)\end{array}$ & UXT (5.48) \\
\hline & 6 & UXT (0.973) & UXT (1.000) & $\begin{array}{l}\text { GAPDH } \\
(0.834)\end{array}$ & АСTB (0.429) & GAPDH (5.96) \\
\hline & 7 & $\begin{array}{l}\text { GAPDH } \\
(1.066)\end{array}$ & $\begin{array}{l}\text { EEF1A1 } \\
(1.120)\end{array}$ & $\begin{array}{l}\text { EEF1A1 } \\
(0.974)\end{array}$ & RPS9 (0.422) & $\begin{array}{l}\text { EEF1A1 } \\
(6.44)\end{array}$ \\
\hline & 8 & RPL4 (1.254) & RPL4 (1.764)) & RPL4 (1.248) & UXT (0.087) & RPL4 (8) \\
\hline & 9 & RPS15 (1.474) & RPS15 (2.015 & RPS15 (1.869) & RPL4 (0.051) & RPS15 (9) \\
\hline & 10 & B2M (1.721) & B2M (2.517) & B2M (2.301) & HPRT1 (0.001) & B2M (10) \\
\hline \multirow{10}{*}{$\begin{array}{l}\text { Double hump } \\
\text { Camel } \\
\text { (DHC) }\end{array}$} & 1 & B2M (0.600) & HPRT1 (0.295) & HPRT1 (0.203) & $\begin{array}{l}\text { GAPDH } \\
(0.372)\end{array}$ & HPRT1 (1.32) \\
\hline & 2 & RPS9 (0.600) & ACTB (0.418) & $\begin{array}{l}\text { EEF1A1 } \\
(0.294)\end{array}$ & АСТВ (0.677) & ACTB (2.63) \\
\hline & 3 & HPRT1 (0.664) & B2M $(0.420)$ & ACTB (0.377) & RPS9 (0.751) & B2M (2.71) \\
\hline & 4 & ACTB (0.680) & RPS9 (0.586) & RPS23 (0.462) & $\begin{array}{l}\text { EEF1A1 } \\
(0.001)\end{array}$ & RPS9 (2.99) \\
\hline & 5 & RPS23 (0.747) & UXT (0.668) & RPS9 (0.542) & RPS15 (0.446) & EEF1A1(4.56) \\
\hline & 6 & $\begin{array}{l}\text { EEF1A1 } \\
(0.794)\end{array}$ & $\begin{array}{l}\text { EEF1A1 } \\
(0.731)\end{array}$ & B2M (0.572) & RPS23 (0.055) & RPS23 (5.60) \\
\hline & 7 & UXT (0.860) & RPS23 (0.915) & UXT (0.693) & UXT (0.774) & UXT (5.92) \\
\hline & 8 & $\begin{array}{l}\text { GAPDH } \\
(0.992)\end{array}$ & RPS15 (1.333) & $\begin{array}{l}\text { GAPDH } \\
(0.862)\end{array}$ & RPL4 (0.599) & GAPDH (8.00) \\
\hline & 9 & RPL4 (1.180) & RPL4 (1.766) & RPL4 (1.538) & B2M (0.809) & RPL4 (9.00) \\
\hline & 10 & RPS15 (1.352) & RPS15 (1.856) & RPS15 (1.61) & HPRT1 (0.797) & $\begin{array}{l}\text { RPS15 } \\
(10.00)\end{array}$ \\
\hline \multirow{2}{*}{$\begin{array}{l}\text { Zanskar } \\
\text { Horse } \\
\text { (ZAP) }\end{array}$} & 1 & RPS9(0.135) & $\begin{array}{l}\text { EEF1A1 } \\
(0.631)\end{array}$ & UXT (0.341) & B2M (0.947) & RPS9 (1.73) \\
\hline & 2 & RPL4 (0.135) & UXT (0.684) & RPS23 (0.434) & ACTB (0.662) & RPL4 (2.51) \\
\hline
\end{tabular}




\begin{tabular}{|lllllll|}
\hline Species & Ranking & geNorm & Normfinder & Bestkeeper & & RefFinder \\
\hline 3 & RPS23 (0.142) & RPS9 (0.749) & RPS9 (0.465) & HPRT1 (0.447) & UXT (2.66) \\
\hline 4 & $\begin{array}{l}\text { EEF1A1 } \\
(0.194)\end{array}$ & RPS23 (0.759) & RPL4 (0.469 & $\begin{array}{l}\text { GAPDH } \\
(0.257)\end{array}$ & $\begin{array}{l}\text { EEF1A1 } \\
(2.78)\end{array}$ \\
\hline 5 & UXT (0.249) & RPL4 (0.763) & $\begin{array}{l}\text { EEF1A1 } \\
(0.498)\end{array}$ & RPS15 (0.182) & RPS23 (3.13) \\
\hline 6 & RPS15 (0.411) & RPS15 (1.022) & RPS15 (0.816) & EEF1A1 & RPS15 (6) \\
\hline 7 & HPRT1 (0.824) & ACTB (1.36) & ACTB (1.211) & RPS9 (0.001) & ACTB (7.24) \\
\hline 8 & ACTB (1.136) & HPRT1 (1.727) & HPRT1 (1.226) & RPS23 (0.001) & HPRT1 (7.74) \\
\hline 9 & B2M (1.394) & B2M (1.945) & B2M (1.914) & UXT (0.001) & B2M (9) \\
\hline 10 & $\begin{array}{l}\text { GAPDH } \\
\text { (1.712) }\end{array}$ & $\begin{array}{l}\text { GAPDH } \\
(2.795)\end{array}$ & $\begin{array}{l}\text { GAPDH } \\
(20.99)\end{array}$ & RPL4 (0.001) & GAPDH (10) \\
\hline
\end{tabular}

The gene expression variation for 10 candidate RGs was also calculated using Best-Keeper algorithm. In BestKeeper analysis, raw Ct values were used to evaluate stability of individual RGs based on their SD and CV values. The lower value indicates higher expression stability; however, the $S D>1$ value indicates the reference gene is unstable and cannot be used for normalization. The RPS15 and GAPDH genes having lowest SD values of $0.123,0.163$ indicated expression stability. This was followed by B2M, EEF1A1, RPL4, UXT, RPS23, RPS9, HPRT1 and ACTB with SD values 0.391, 0.468, 0.481, 0.491, 0.503, 0.642, 0.712 and 0.812 , respectively (Table 4). The ACTB gene on the other hand was least stable gene with highest SD value. Additionally, the inter-gene relationship for 10 RGs pairs was also estimated. Strong correlation coefficients ( $r$ ) were observed for RPL4/EEF1A1 (0.980), EEF1A1/RPS9 (0.971), RPS23/RPS9 (0.966), RPS23/EEF1A1 (0.962), RPL4/RPS23 (0.961), RPL4/ RPS9 (0.922), UXT/ RPS9 (0.898) (Table 5). This analysis provided strong evidence that these pair of genes have similar expression pattern across the animals. Further Best-Keeper index was calculated for each gene and the correlation between each candidate RGs and Best-Keeper was estimated. The relationship between RGs and Best-Keeper was described in terms of Pearson correlation coefficient ( $r$ ), coefficient of determination $\left(r^{2}\right)$ and the $p$ value. The $p<0.05$ was obtained for all genes indicating a significant contribution of all genes towards the index. Though the EEF1A1 (0.978) and RPS9 (0.973) showed high correlation values but their high fold change makes these genes as unreliable reference genes. The statistically significant SD and correlation shown by the RGs from with BestKeeper algorithm appeared to be consistent with their evaluation assessed by geNorm and Normfinder. 
Table 4

Analysis of parameters based quantitative cycling points (CP) for 10 candidate RGs in LAC

\begin{tabular}{|c|c|c|c|c|c|c|c|c|c|c|}
\hline & GAPDH & ACTB & RPS9 & EEF1A1 & RPS15 & RPS23 & $U X T$ & RPL4 & $B 2 M$ & HPRT1 \\
\hline $\mathrm{n}$ & 6 & 6 & 6 & 6 & 6 & 6 & 6 & 6 & 6 & 6 \\
\hline geo Mean [CP] & 22.170 & 23.810 & 20.460 & 17.420 & 19.510 & 14.410 & 24.630 & 20.120 & 18.550 & 28.860 \\
\hline AR Mean [CP] & 22.170 & 23.830 & 20.480 & 17.440 & 19.510 & 14.420 & 24.640 & 20.130 & 18.560 & 28.870 \\
\hline $\min [\mathrm{CP}]$ & 21.870 & 22.640 & 19.870 & 16.900 & 19.210 & 13.940 & 23.860 & 19.610 & 17.740 & 27.950 \\
\hline $\max [\mathrm{CP}]$ & 22.530 & 24.900 & 22.400 & 18.810 & 19.790 & 15.930 & 26.070 & 21.560 & 19.360 & 30.180 \\
\hline std dev [+/- CP] & 0.16 & 0.81 & 0.64 & 0.46 & 0.12 & 0.50 & 0.49 & 0.48 & 0.39 & 0.71 \\
\hline $\mathrm{CV}[\% \mathrm{CP}]$ & 0.740 & 3.410 & 3.130 & 2.630 & 0.630 & 3.490 & 1.980 & 2.390 & 2.110 & 2.470 \\
\hline $\min [x$-fold $]$ & -1.230 & -2.250 & -1.500 & -1.440 & -1.230 & -1.380 & -1.710 & -1.430 & -1.750 & -1.870 \\
\hline $\max [x$-fold $]$ & 1.280 & 2.130 & 3.840 & 2.610 & 1.210 & 2.880 & 2.710 & 2.710 & 1.750 & 2.500 \\
\hline $\begin{array}{l}\text { std dev [+/- } x- \\
\text { fold] }\end{array}$ & 1.120 & 1.760 & 1.560 & 1.370 & 1.090 & 1.420 & 1.400 & 1.400 & 1.310 & 1.640 \\
\hline $\begin{array}{l}\mathrm{N}=\text { number of } \mathrm{s} \\
{[\mathrm{CP}]=\text { extreme }} \\
\text { a percentage or } \\
\mathrm{x} \text {-fold over or ur }\end{array}$ & $\begin{array}{l}\text { of of } \mathrm{CP} \\
\mathrm{CP} \text { val } \\
\text { oeffic }\end{array}$ & in [ & $\mathrm{d}$ & fold & - & 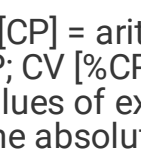 & ocio & t of & on & $\begin{array}{l}\mathrm{d} \text { max } \\
\text { ssed as } \\
\text { solute }\end{array}$ \\
\hline
\end{tabular}


Table 5

Analysis of repeated pair-wise correlation amongst genes in LAC with BestKeeper index

\begin{tabular}{|c|c|c|c|c|c|c|c|c|c|c|}
\hline & GAPDH & ACTB & RPS9 & EEF1A1 & RPS15 & RPS23 & $U X T$ & RPL4 & $B 2 M$ & HPRT1 \\
\hline ACTB & -0.107 & - & - & - & - & - & - & - & - & - \\
\hline p-value & 0.84 & - & - & - & - & - & - & - & - & - \\
\hline RPS9 & -0.08 & 0.309 & - & - & - & - & - & - & - & - \\
\hline$p$-value & 0.88 & 0.552 & - & - & - & - & - & - & - & - \\
\hline EEF1A1 & -0.056 & 0.316 & 0.971 & - & - & - & - & - & - & - \\
\hline p-value & 0.916 & 0.542 & 0.001 & - & - & - & - & - & - & - \\
\hline RPS15 & -0.856 & 0.072 & -0.014 & 0.08 & - & - & - & - & - & - \\
\hline$p$-value & 0.03 & 0.892 & 0.979 & 0.881 & - & - & - & - & - & - \\
\hline RPS23 & -0.14 & 0.252 & 0.966 & 0.962 & 0.137 & - & - & - & - & - \\
\hline p-value & 0.791 & 0.631 & 0.002 & 0.002 & 0.796 & - & - & - & - & - \\
\hline$U X T$ & 0.339 & 0.378 & 0.898 & 0.888 & -0.344 & 0.849 & - & - & - & - \\
\hline$p$-value & 0.511 & 0.46 & 0.015 & 0.018 & 0.504 & 0.033 & - & - & - & - \\
\hline RPL4 & -0.102 & 0.34 & 0.922 & 0.98 & 0.206 & 0.961 & 0.84 & - & - & - \\
\hline$p$-value & 0.848 & 0.51 & 0.009 & 0.001 & 0.695 & 0.002 & 0.036 & - & - & - \\
\hline$B 2 M$ & 0.396 & -0.148 & 0.585 & 0.611 & -0.197 & 0.698 & 0.702 & 0.652 & - & - \\
\hline p-value & 0.436 & 0.779 & 0.222 & 0.197 & 0.709 & 0.123 & 0.12 & 0.16 & - & - \\
\hline HPRT1 & 0.052 & 0.4 & 0.82 & 0.747 & -0.317 & 0.645 & 0.775 & 0.612 & 0.162 & - \\
\hline$p$-value & 0.922 & 0.432 & 0.046 & 0.088 & 0.541 & 0.166 & 0.07 & 0.197 & 0.759 & - \\
\hline BestKeeper vs. & GAPDH & ACTB & RPS9 & EEF1A1 & RPS15 & RPS23 & UXT & RPL4 & $B 2 M$ & HPRT1 \\
\hline coeff. of corr. [r] & 0.017 & 0.433 & 0.973 & 0.978 & 0.001 & 0.959 & 0.941 & 0.959 & 0.637 & 0.763 \\
\hline p-value & 0.975 & 0.392 & 0.001 & 0.001 & 0.971 & 0.003 & 0.005 & 0.003 & 0.173 & 0.078 \\
\hline
\end{tabular}

Additionally, RefFinder based analysis was carried out that ranks the stability order of RGs in a more refined way by taking into consideration geNorm, NormFinder, BestKeeper, and delta Ct algorithms. The stability order and ranking of the RGs as per RefFinder were; EEF1A1 (1.41) RPL4 (2.11), RPS23 (3.98), UXT (4.36), RPS15 (4.76), B2M (5.05), GAPDH(5.12), RPS9 (5.32), HPRT1 (9), ACTB (10).

\section{Ladakhi Yak (LAY)}

The $\mathrm{M}$ value for all the 10 genes in geNorm analysis were found to be within acceptable range in LAY. The ranking order of RGs was GAPDH=RPS9>ACTB>RPS23>HPRT1>UXT>B2M>EEF1A1>RPL4>RPS15 (Fig. 2d). GAPDH and RPS9 showed higher gene expression stability with $\mathrm{M}$ value of 0.223 followed by ACTB, RPS23 and HPRT1 with $\mathrm{M}$ value of $0.386,0.507$, 0.595 respectively (Table 3). On the other hand, RPS15, RPL4 and EEF1A1 were least stable with higher M values of 1.242, 1.121 and 0.992 , respectively. The pair wise variation analysis showed $V 4 / 5$ combination with least $V$ value (0.132) followed by V3/4 (0.148) and V5/V6 (0.150) combinations (Fig. 2e). Since all these V values were well within the acceptable range (recommended cut-off value 0.15 ), therefore use of panel of $3 \mathrm{RGs}$ (GAPDH, RPS9 and ACTB) is likely to provide most accurate normalization in Ladakhi yak samples. The Normfinder analysis also identified same set of RGs in LAY samples with 
highest stability; GAPDH(0.112), RPS9 (0.112) and $A C T B(0.202)$ albeit slight change in their ranking order; GAPDH> RPS9> $A C T B>R P S 23>H P R T 1>U X T>E E F 1 A 1>B 2 M>R P S 15>R P L 4$ (Fig. 2f \& Table 3). Similar to geNorm, RPL4 (1.538) and RPS15 (1.523) were found to be least stable RGs.

In BestKeeper analysis, UXT was found to be most stable with minimum SD value (0.180) followed by HPRT1, RPL4, GAPDH, $A C T B, R P S 9, R P S 23, B 2 M, E E F 1 A 1, R P S 15$ with the SD values of $0.240,0.500,0.600,0.610,0.680,0.840,1.350,1.590,1.750$, respectively (Table 6). Additionally, high correlation coefficient was observed for RPS9/GAPDH $(\mathrm{r}=0.973), B 2 M / A C T B$ $(\mathrm{r}=0.942), E E F 1 A 1 / G A P D H(\mathrm{r}=0.931), E E F 1 A 1 / R P S 9(\mathrm{r}=0.923), R P S 23 / A C T B(\mathrm{r}=0.914)$ and B2M/EEF1A1 $(\mathrm{r}=0.909)$ pair combinations. The best correlation between RGs and BestKeeper was observed for GAPDH ( $\mathrm{r}=0.967)$, RPS9 ( $\mathrm{r}=0.965), E E F 1 A 1$ $(\mathrm{r}=0.960), A C T B(\mathrm{r}=0.929), B 2 M(\mathrm{r}=0.927)$ (Table 7). The high correlation values for these genes indicated their reliability as RGs, The GAPDH, RPS9 and ACTB were termed as best RGs on the basis of highest correlation value and less SD.

Table 6

Analysis of parameters based quantitative cycling points (CP) for 10 candidate RGs in LAY

\begin{tabular}{|lllllllllll|}
\hline & GAPDH & ACTB & RPS9 & EEF1A1 & RPS15 & RPS23 & UXT & RPL4 & B2M & HPRT1 \\
\hline $\mathrm{n}$ & 6 & 6 & 6 & 6 & 6 & 6 & 6 & 6 & 6 & 6 \\
\hline geo Mean [CP] & 18.29 & 20.97 & 22.18 & 22.16 & 31.87 & 16.13 & 22.76 & 24.31 & 21.44 & 24.92 \\
\hline AR Mean [CP] & 18.31 & 20.98 & 22.2 & 22.23 & 31.93 & 16.16 & 22.76 & 24.32 & 21.51 & 24.93 \\
\hline min [CP] & 17.34 & 19.62 & 21.06 & 19.57 & 29.06 & 14.37 & 22.22 & 23.71 & 18.11 & 24.33 \\
\hline max [CP] & 19.2 & 22.12 & 23.42 & 23.62 & 33.82 & 17.51 & 23.04 & 25.65 & 22.95 & 25.41 \\
\hline std dev [+/- CP] & 0.60 & 0.61 & 0.68 & 1.59 & 1.75 & 0.84 & 0.18 & 0.50 & 1.35 & 0.24 \\
\hline CV [\% CP] & 3.3 & 2.9 & 3.06 & 7.14 & 5.47 & 5.21 & 0.8 & 2.06 & 6.29 & 0.98 \\
\hline min [x-fold] & -1.94 & -2.54 & -2.18 & -6.02 & -7.04 & -3.39 & -1.46 & -1.52 & -10.08 & -1.51 \\
\hline max [x-fold] & 1.88 & 2.22 & 2.36 & 2.75 & 3.85 & 2.6 & 1.21 & 2.53 & 2.84 & 1.4 \\
\hline std dev [+/- x-fold] & 1.52 & 1.52 & 1.6 & 3.01 & 3.35 & 1.79 & 1.13 & 1.42 & 2.55 & 1.18 \\
\hline
\end{tabular}


Table 7

Analysis of repeated pair-wise correlation amongst genes in LAY with BestKeeper index

\begin{tabular}{|c|c|c|c|c|c|c|c|c|c|c|}
\hline & GAPDH & ACTB & RPS9 & EEF1A1 & RPS15 & RPS23 & $U X T$ & RPL4 & $B 2 M$ & HPRT1 \\
\hline ACTB & 0.859 & - & - & - & - & - & - & - & - & - \\
\hline p-value & 0.028 & - & - & - & - & - & - & - & - & - \\
\hline RPS9 & 0.973 & 0.84 & - & - & - & - & - & - & - & - \\
\hline p-value & 0.001 & 0.037 & - & - & - & - & - & - & - & - \\
\hline EEF1A1 & 0.931 & 0.887 & 0.923 & - & - & - & - & - & - & - \\
\hline p-value & 0.007 & 0.019 & 0.009 & - & - & - & - & - & - & - \\
\hline RPS15 & 0.892 & 0.632 & 0.805 & 0.751 & - & - & - & - & - & - \\
\hline p-value & 0.017 & 0.178 & 0.053 & 0.085 & - & - & - & - & - & - \\
\hline RPS23 & 0.784 & 0.914 & 0.755 & 0.786 & 0.693 & - & - & - & - & - \\
\hline p-value & 0.065 & 0.011 & 0.083 & 0.064 & 0.127 & - & - & - & - & - \\
\hline$U X T$ & -0.437 & -0.581 & -0.265 & -0.31 & -0.525 & -0.652 & - & - & - & - \\
\hline p-value & 0.386 & 0.227 & 0.611 & 0.55 & 0.285 & 0.161 & - & - & - & - \\
\hline RPL4 & -0.565 & -0.792 & -0.597 & -0.799 & -0.322 & -0.761 & 0.207 & - & - & - \\
\hline p-value & 0.243 & 0.06 & 0.211 & 0.056 & 0.533 & 0.079 & 0.694 & - & - & - \\
\hline$B 2 M$ & 0.82 & 0.942 & 0.87 & 0.909 & 0.538 & 0.858 & -0.292 & -0.879 & - & - \\
\hline p-value & 0.046 & 0.005 & 0.024 & 0.012 & 0.271 & 0.029 & 0.574 & 0.021 & - & - \\
\hline HPRT1 & 0.62 & 0.602 & 0.777 & 0.671 & 0.371 & 0.587 & 0.186 & -0.634 & 0.81 & - \\
\hline p-value & 0.19 & 0.206 & 0.069 & 0.145 & 0.47 & 0.221 & 0.724 & 0.176 & 0.051 & - \\
\hline BestKeeper vs. & GAPDH & ACTB & RPS9 & EEF1A1 & RPS15 & RPS23 & $U X T$ & $R P L 4$ & $B 2 M$ & HPRT1 \\
\hline coeff. of corr. [r] & 0.967 & 0.929 & 0.965 & 0.96 & 0.813 & 0.886 & 0.001 & 0.001 & 0.927 & 0.727 \\
\hline p-value & 0.002 & 0.007 & 0.002 & 0.002 & 0.049 & 0.019 & 0.401 & 0.096 & 0.008 & 0.102 \\
\hline
\end{tabular}

The RefFinder analysis also identified GAPDH, RPS9 and ACTB to be most stable RGs while RPS15, B2M and EEF1A1 were the least stable RGS in LAY. In the present investigation, all four methods geNorm, Normfinder BestKeeper and RefFinder have demonstrated that GAPDH, RPS9 and ACTB are the most stable RGs in PBMCs of LAY.

\section{Ladakhi Donkey (LAD)}

In Ladakhi donkey as well, the geNorm analysis showed mean expression stability values of 10 RGs within the acceptable range and varied from $0.250(H P R T 1=B 2 M)$ to $1.405(R P S 9)$ (Table 3). The stability ranking of RGs was: HPRT1=B2M> RPS23>ACTB> EEF1A1> GAPDH>UXT>RPL4> RPS15> RPS9 (Fig. 2g). The B2M and HPRT1 RGs showed highest expression stability with lowest $\mathrm{M}$ value while RPS9 and RPS15RGs showed least expression stability with highest $\mathrm{M}$ value. Based on pair-wise variation analysis (V value), V3/4 combination (B2M HPRT1 and RPS23) with $\mathrm{V}$ value of 0.142 was found to provide the most accurate normalization in Ladakhi donkey (Fig. 2h). In Normfinder analysis as well; HPRT1 (0.123), B2M (0.324) and ACTB (0.518) were most stable with lowest values (Fig. 2i). On the other hand, the RPS9 (1.912), RPS15 (1.377), and RPL4 (1.366) RGs on the other hand were least stable. 
The BestKeeper analysis showed ACTB gene to be most stable with the lowest crossing point SD value of 0.295 . This was followed by HPRT1, RPS23 and B2M RGs with SD value of $0.311,0.318$, and 0.472 , respectively. On the other hand, $R P S 9$ with highest crossing point SD value of 1.635 was found to be the least stable (Table 8). In addition, the inter-gene relation for 10 RGs pairs was also estimated. B2M/GAPDH ( $\mathrm{r}=1.0), H P R T 1 / B 2 M(\mathrm{r}=0.985), H P R T 1 / G A P D H(\mathrm{r}=0.985), B 2 M / E E F 1 A 1(\mathrm{r}=0.855)$ and EEF1A1/GAPDH $(\mathrm{r}=0.854)$ showed the strong correlation coefficients (Table 9). The highly correlated RGs were combined into BestKeeper index, and the correlation between each candidate RGs and BestKeeper was estimated. The relationship between RG and BestKeeper was described in terms of Pearson correlation coefficient ( $r$ ), coefficient of determination correlation between BestKeeper and RGs was observed for HPRT1 ( $\mathrm{r}=0.942)$ and GAPDH ( $\mathrm{r}=0.941)$ followed by B2M (0.940) and EEF1A1 (0.927) genes. The statistically significant correlation shown by RGs (HPRT1, B2M) with the BestKeeper index appeared to be consistent with their evaluation as assessed by geNorm and Normfinder. RefFinder was another tool, were evaluating and identified RGs from comprehensive data set. HPRT1, B2M and ACTB were most stable and RPS9, RPS15 and GAPDH were least stable genes identified by RefFinder in LAD.

Table 8

Analysis of parameters based quantitative cycling points (CP) for 10 candidate RGs in LAD

\begin{tabular}{|lllllllllll|}
\hline & GAPDH & ACTB & RPS9 & EEF1A1 & RPS15 & RPS23 & UXT & RPL4 & B2M & HPRT1 \\
\hline $\mathrm{n}$ & 5 & 5 & 5 & 5 & 5 & 5 & 5 & 5 & 5 & 5 \\
\hline geo Mean [CP] & 22.83 & 20.66 & 21.35 & 19.45 & 33.76 & 14.33 & 22.69 & 21.66 & 17.74 & 25.56 \\
\hline AR Mean [CP] & 22.87 & 20.66 & 21.43 & 19.48 & 33.78 & 14.34 & 22.71 & 21.68 & 17.75 & 25.57 \\
\hline min [CP] & 20.73 & 19.97 & 19.7 & 17.94 & 31.86 & 13.86 & 21.45 & 20.55 & 16.69 & 24.79 \\
\hline max [CP] & 24.53 & 21.09 & 24.16 & 20.65 & 35.47 & 14.84 & 23.92 & 23.17 & 18.58 & 26.04 \\
\hline std dev [+/- CP] & 0.95 & 0.30 & 1.64 & 0.85 & 0.92 & 0.32 & 0.61 & 0.60 & 0.47 & 0.31 \\
\hline CV [\% CP] & 4.16 & 1.43 & 7.63 & 4.37 & 2.72 & 2.22 & 2.7 & 2.76 & 2.66 & 1.22 \\
\hline min [x-fold] & -4.3 & -1.61 & -3.15 & -2.86 & -3.73 & -1.39 & -2.36 & -2.16 & -2.07 & -1.71 \\
\hline max [x-fold] & 3.24 & 1.35 & 6.99 & 2.29 & 3.27 & 1.42 & 2.34 & 2.85 & 1.79 & 1.39 \\
\hline std dev [+/- x-fold] & 1.93 & 1.23 & 3.11 & 1.81 & 1.89 & 1.25 & 1.53 & 1.51 & 1.39 & 1.24 \\
\hline
\end{tabular}


Table 9

Analysis of repeated pair-wise correlation amongst genes in LAD with BestKeeper index

\begin{tabular}{|c|c|c|c|c|c|c|c|c|c|c|}
\hline & GAPDH & ACTB & RPS9 & EEF1A1 & RPS15 & RPS23 & $U X T$ & RPL4 & $B 2 M$ & HPRT1 \\
\hline ACTB & -0.067 & - & - & - & - & - & - & - & - & - \\
\hline p-value & 0.915 & - & - & - & - & - & - & - & - & - \\
\hline RPS9 & 0.345 & -0.434 & - & - & - & - & - & - & - & - \\
\hline p-value & 0.57 & 0.465 & - & - & - & - & - & - & - & - \\
\hline EEF1A1 & 0.854 & 0.096 & 0.608 & - & - & - & - & - & - & - \\
\hline $\mathrm{p}$-value & 0.065 & 0.878 & 0.276 & - & - & - & - & - & - & - \\
\hline RPS15 & -0.301 & -0.134 & 0.629 & -0.036 & - & - & - & - & - & - \\
\hline p-value & 0.623 & 0.83 & 0.256 & 0.954 & - & - & - & - & - & - \\
\hline RPS23 & -0.004 & 0.363 & -0.793 & -0.171 & -0.931 & - & - & - & - & - \\
\hline $\mathrm{p}$-value & 0.995 & 0.548 & 0.109 & 0.783 & 0.022 & - & - & - & - & - \\
\hline$U X T$ & -0.281 & 0.204 & -0.827 & -0.674 & -0.289 & 0.372 & - & - & - & - \\
\hline $\mathrm{p}$-value & 0.647 & 0.742 & 0.084 & 0.212 & 0.638 & 0.538 & - & - & - & - \\
\hline RPL4 & -0.71 & -0.162 & -0.709 & -0.895 & -0.353 & 0.51 & 0.581 & - & - & - \\
\hline $\mathrm{p}$-value & 0.179 & 0.795 & 0.18 & 0.04 & 0.56 & 0.381 & 0.304 & - & - & - \\
\hline$B 2 M$ & 1 & -0.065 & 0.343 & 0.855 & -0.304 & 0 & -0.282 & -0.709 & - & - \\
\hline$p$-value & 0.001 & 0.918 & 0.572 & 0.065 & 0.619 & 1 & 0.646 & 0.18 & - & - \\
\hline HPRT1 & 0.985 & -0.04 & 0.339 & 0.814 & -0.206 & -0.094 & -0.191 & -0.739 & 0.985 & - \\
\hline $\mathrm{p}$-value & 0.002 & 0.949 & 0.577 & 0.094 & 0.74 & 0.881 & 0.758 & 0.153 & 0.002 & - \\
\hline BestKeeper vs. & GAPDH & ACTB & RPS9 & EEF1A1 & RPS15 & RPS23 & UXT & RPL4 & $B 2 M$ & HPRT1 \\
\hline coeff. of corr. [r] & 0.941 & 0.001 & 0.619 & 0.927 & 0.022 & 0.001 & 0.001 & 0.001 & 0.94 & 0.942 \\
\hline p-value & 0.017 & 0.863 & 0.265 & 0.024 & 0.972 & 0.612 & 0.406 & 0.046 & 0.018 & 0.017 \\
\hline
\end{tabular}

\section{Chanthangi Goat (CHG)}

The geNorm analysis of all the 10 candidate RGs in Changthangi goat exhibited mean expression stability (M) values well below 1.5 (Table 3). The stability ranking RGs were in the following order; RPS9=HPRT $>A C T B>R P S 23>E E F 1 A 1>U X T$ $>G A P D H>R P L 4>R P S 15>B 2 M$ (Fig. 2j). The RPS9 and HPRT were most stable with lowest $\mathrm{M}$ value of 0.378 while RPS 15 and $B 2 M$ had maximum expression variability and highest $M$ values of 1.474 and 1.721 , respectively.

Further, the pair-wise variation analysis provided within the acceptable limit on sequential addition of another gene to the two most stably expressed genes, viz., B2M and HPRT1, the pair-wise combination V2/3 gave the acceptable $\mathrm{V}$ value of 0.143 $(<0.15)$ suggesting that the geometric mean between RPS9, HPRT1 and ACTB is optimal for data normalization in Changthangi goat (Fig. 2k). Similar to geNorm, Normfinder also identified RPS9 (0.310), RPS23 (0.477), ACTB (0.486) and HPRT1 (0.740) as most stable and B2M (2.517) and RPS15(2.015) as least stably expressed genes (Fig. 2l \& Table 3). There was good agreement between geNorm and Normfinder outcome, albeit slight variation was observed in the ranking of RGs. The BestKeeper algorithm showed consistent expression levels for all the RGs. RPS9 (0.176), exhibited low SD and 0.422 
correlation coefficients in BestKeeper analysis, pointing towards their expression stability (Table 10). Additionally, RPS9/ACTB $(\mathrm{r}=0.974), B 2 M / R P S 23(\mathrm{r}=0.801), B 2 M / E E F 1 A 1$ ( $\mathrm{r}=0.739)$, and RPS23/GAPDH $(\mathrm{r}=0.712)$ showed the strong correlation coefficients (Table 11). B2M (0.847) showed the high correlation value but they showed the high fold change thus their reliability as a RGs is not applicable. RefFinder were identified the overall ranking of the gene. The ranking of genes was $R P S 9$ (1), HPRT1 (2.38), ACTB (2.71), RPS23 (3.13), UXT (5.48), GAPDH(5.96), EEF1A1 (6.44), RPL4 (8), RPS15 (9), B2M (10). In the present investigation, all four algorithmic methods geNorm Normfinder, BestKeeper and RefFinder have demonstrated that RPS9, HPRT1 and ACTB are the most stable RGs in CHG.

Table 10

Analysis of parameters based quantitative cycling points (CP) for 10 candidate RGs in CHG

\begin{tabular}{|lllllllllll|l|}
\hline & GAPDH & ACTB & RPS9 & EEF1A1 & RPS15 & RPS23 & UXT & RPL4 & B2M & HPRT1 \\
\hline $\mathrm{n}$ & 5 & 5 & 5 & 5 & 5 & 5 & 5 & 5 & 5 & 5 \\
\hline geo Mean [CP] & 15.8 & 17.92 & 19.46 & 19.09 & 32.82 & 14.37 & 21.39 & 21.92 & 30.29 & 24.86 \\
\hline AR Mean [CP] & 15.84 & 17.93 & 19.46 & 19.12 & 32.88 & 14.39 & 21.41 & 21.97 & 30.39 & 24.86 \\
\hline min [CP] & 14.35 & 17.09 & 19.15 & 17.75 & 30.18 & 13.63 & 20.44 & 20.01 & 26.7 & 24.36 \\
\hline max [CP] & 17.4 & 18.64 & 19.79 & 20.64 & 34.92 & 15.23 & 22.62 & 23.87 & 33.05 & 25.45 \\
\hline std dev [+/- CP] & 0.83 & 0.49 & 0.18 & 0.97 & 1.87 & 0.6 & 0.63 & 1.25 & 2.3 & 0.32 \\
\hline CV [\% CP] & 5.27 & 2.71 & 0.9 & 5.1 & 5.68 & 4.14 & 2.96 & 5.68 & 7.57 & 1.3 \\
\hline min [x-fold] & -2.74 & -1.78 & -1.24 & -2.54 & -6.22 & -1.67 & -1.94 & -3.77 & -12 & -1.41 \\
\hline max [x-fold] & 3.02 & 1.64 & 1.26 & 2.92 & 4.3 & 1.81 & 2.34 & 3.85 & 6.8 & 1.51 \\
\hline std dev [+/- x-fold] & 1.78 & 1.4 & 1.13 & 1.96 & 3.65 & 1.51 & 1.55 & 2.38 & 4.93 & 1.25 \\
\hline
\end{tabular}


Table 11

Analysis of repeated pair-wise correlation amongst genes in $\mathrm{CHG}$ with BestKeeper index

\begin{tabular}{|c|c|c|c|c|c|c|c|c|c|c|}
\hline & GAPDH & ACTB & RPS9 & EEF1A1 & RPS15 & RPS23 & $U X T$ & RPL4 & $B 2 M$ & HPRT1 \\
\hline ACTB & -0.379 & - & - & - & - & - & - & - & - & - \\
\hline p-value & 0.53 & - & - & - & - & - & - & - & - & - \\
\hline RPS9 & -0.431 & 0.974 & - & - & - & - & - & - & - & - \\
\hline$p$-value & 0.468 & 0.005 & - & - & - & - & - & - & - & - \\
\hline EEF1A1 & 0.096 & 0.663 & 0.482 & - & - & - & - & - & - & - \\
\hline $\mathrm{p}$-value & 0.878 & 0.222 & 0.412 & - & - & - & - & - & - & - \\
\hline RPS15 & 0.163 & 0.422 & 0.522 & -0.041 & - & - & - & - & - & - \\
\hline$p$-value & 0.793 & 0.479 & 0.367 & 0.948 & - & - & - & - & - & - \\
\hline RPS23 & 0.712 & -0.035 & -0.11 & 0.398 & -0.185 & - & - & - & - & - \\
\hline$p$-value & 0.177 & 0.956 & 0.86 & 0.507 & 0.766 & - & - & - & - & - \\
\hline$U X T$ & 0.341 & -0.574 & -0.393 & -0.852 & 0.335 & -0.009 & - & - & - & - \\
\hline $\mathrm{p}$-value & 0.574 & 0.311 & 0.513 & 0.067 & 0.581 & 0.988 & - & - & - & - \\
\hline RPL4 & -0.293 & 0.05 & 0.274 & -0.673 & 0.441 & -0.264 & 0.689 & - & - & - \\
\hline$p$-value & 0.632 & 0.937 & 0.655 & 0.213 & 0.457 & 0.668 & 0.199 & - & - & - \\
\hline$B 2 M$ & 0.697 & 0.334 & 0.208 & 0.739 & 0.194 & 0.801 & -0.292 & -0.475 & - & - \\
\hline$p$-value & 0.191 & 0.583 & 0.737 & 0.154 & 0.754 & 0.103 & 0.633 & 0.419 & - & - \\
\hline HPRT 1 & -0.625 & 0.533 & 0.478 & 0.456 & -0.494 & 0.081 & -0.669 & -0.08 & -0.034 & - \\
\hline p-value & 0.26 & 0.355 & 0.415 & 0.44 & 0.398 & 0.897 & 0.217 & 0.898 & 0.956 & - \\
\hline BestKeeper vs. & GAPDH & ACTB & RPS9 & EEF1A1 & RPS15 & RPS23 & $U X T$ & RPL4 & $B 2 M$ & HPRT1 \\
\hline coeff. of corr. [r] & 0.623 & 0.429 & 0.422 & 0.435 & 0.58 & 0.676 & 0.087 & 0.051 & 0.847 & 0.001 \\
\hline$p$-value & 0.262 & 0.471 & 0.479 & 0.464 & 0.305 & 0.211 & 0.89 & 0.935 & 0.07 & 0.783 \\
\hline
\end{tabular}

\section{Double hump Camel (DHC)}

The geNorm analysis of 10 RGs showed M values ranging from 0.600 to 1.352 in double hump camel (Table 3 ). The $M$ values for all the RGs were within the acceptable limit of $<1.5$. On the basis of relative expression stability and stepwise exclusion, the ranking order of RGs was: $B 2 M=R P S 9>H P R T 1>A C T B>R P S 23>E E F 1 A 1>U X T>G A P D H>R P L 4>R P S 15$ (Fig. $2 \mathrm{~m})$. The expression of RPS9 and B2MRGs with lowest M values of 0.600 were found to be most stable while RPL4 and RPS15RGs with highest $M$ values of 1.180 and 1.352, respectively were found to be least stable RGs in DHC. Based on pair-wise combination, the $\mathrm{V}$ values for $\mathrm{V} 3 / 4, \mathrm{~V} 5 / 6$ and $\mathrm{V} 6 / 7$ and were close to the threshold value of 0.15 . Therefore, the combination of V3/4 with ACTB, HPRT1 and B2M RGs should provide the accurate normalization of qPCR data in DHC (Fig. 2n).

In Normfinder analysis, the RGs were ranked as follows: HPRT1>ACTB>B2M>RPS9>UXT>EEF1A1>RPS23>GAPDH>RPL4> RPS15 (Fig. 20). The HPRT1 (0.295), ACTB (0.418), B2M (0.420), RPS9 (0.586) were four most stable RGs as per stability values. 
In BestKeeper analysis, HPRT1 gene with the lowest crossing point SD value of 0.203 was found to be most stable. This was followed by EEF1A1, ACTB and RPS23 genes with SD values of $0.294,0.377$, and 0.462 , respectively (Table12). On the other hand, RPS15, RPL4 and GAPDHRGs with high crossing point SD values of 1.61, 1.54, 0.86 respectively were found to be least stable. Strong correlation was observed in inter gene relationship of the RGs RPL4/UXT ( $\mathrm{r}=0.908), H P R T 1 / U X T(\mathrm{r}=0.884)$ and HPRT1/B2M ( $\mathrm{r}=0.755)$ (Table 13). The relationship between RGs and BestKeeper was described in terms of Pearson correlation coefficient ( $\mathrm{r}$ ), coefficient of determination correlation between BestKeeper and RGs was observed for HPRT1 $(\mathrm{r}=0.797)$ and $B 2 M(\mathrm{r}=0.809)$ followed by UXT, RPS9 and ACTB gene.

ReFinder based overall analysis resulted in stability ranking of RGs as; HPRT1 (1.32) > ACTB (2.63) >, B2M (2.71) >, RPS9 (2.99) > EEF1A1, RPS23 (5.60) >UXT (5.92)> GAPDH (8.00)> RPL4 (9.00)> RPS15 (10.00). Overall, HPRT1, B2M and ACTB were identified as the most appropriate RGs in high altitude adapted DHC using all four algorithms.

Table 12

Analysis of parameters based quantitative cycling points (CP) for 10 candidate RGs in DHC

\begin{tabular}{|llllllllllll|}
\hline & GAPDH & ACTB & RPS9 & EEF1A1 & RPS15 & RPS23 & UXT & RPL4 & B2M & HPRT1 \\
\hline $\mathrm{n}$ & 5 & 5 & 5 & 5 & 5 & 5 & 5 & 5 & 5 & 5 \\
\hline geo Mean [CP] & 22.53 & 19.15 & 19.57 & 17.38 & 32.37 & 17.04 & 19.66 & 20.31 & 20.41 & 22.27 \\
\hline AR Mean [CP] & 22.57 & 19.16 & 19.58 & 17.39 & 32.42 & 17.05 & 19.68 & 20.38 & 20.42 & 22.27 \\
\hline min [CP] & 20.41 & 18.37 & 18.55 & 16.87 & 30.62 & 16.05 & 18.19 & 18.23 & 19.24 & 21.93 \\
\hline max [CP] & 24.13 & 20 & 20.83 & 17.93 & 34.9 & 17.98 & 20.89 & 22.32 & 21.4 & 22.78 \\
\hline std dev [+/- CP] & 0.86 & 0.38 & 0.54 & 0.29 & 1.61 & 0.46 & 0.69 & 1.54 & 0.57 & 0.2 \\
\hline CV [\% CP] & 3.82 & 1.97 & 2.77 & 1.69 & 4.97 & 2.71 & 3.52 & 7.55 & 2.8 & 0.91 \\
\hline min [x-fold] & -4.35 & -1.72 & -2.03 & -1.43 & -3.37 & -1.98 & -2.76 & -4.23 & -2.25 & -1.27 \\
\hline max [x-fold] & 3.03 & 1.8 & 2.4 & 1.46 & 5.77 & 1.92 & 2.35 & 4.03 & 1.99 & 1.42 \\
\hline std dev [+/- x-fold] & 1.82 & 1.3 & 1.46 & 1.23 & 3.05 & 1.38 & 1.62 & 2.9 & 1.49 & 1.15 \\
\hline
\end{tabular}


Table 13

Analysis of repeated pair-wise correlation amongst genes in DHC with BestKeeper index

\begin{tabular}{|c|c|c|c|c|c|c|c|c|c|c|}
\hline & GAPDH & ACTB & RPS9 & EEF1A1 & RPS15 & RPS23 & $U X T$ & RPL4 & $B 2 M$ & HPRT1 \\
\hline ACTB & -0.075 & - & - & - & - & - & - & - & - & - \\
\hline p-value & 0.905 & - & - & - & - & - & - & - & - & - \\
\hline RPS9 & 0.471 & 0.665 & - & - & - & - & - & - & - & - \\
\hline p-value & 0.423 & 0.22 & - & - & - & - & - & - & - & - \\
\hline EEF1A1 & 0.42 & -0.536 & -0.552 & - & - & - & - & - & - & - \\
\hline p-value & 0.482 & 0.352 & 0.334 & - & - & - & - & - & - & - \\
\hline RPS15 & 0.383 & -0.043 & -0.155 & 0.519 & - & - & - & - & - & - \\
\hline p-value & 0.524 & 0.946 & 0.804 & 0.37 & - & - & - & - & - & - \\
\hline RPS23 & 0.194 & 0.591 & 0.528 & -0.129 & -0.48 & - & - & - & - & - \\
\hline$p$-value & 0.754 & 0.294 & 0.36 & 0.836 & 0.413 & - & - & - & - & - \\
\hline$U X T$ & -0.247 & 0.571 & 0.324 & -0.569 & 0.386 & -0.318 & - & - & - & - \\
\hline p-value & 0.689 & 0.315 & 0.594 & 0.317 & 0.521 & 0.602 & - & - & - & - \\
\hline$R P L 4$ & -0.471 & 0.569 & 0.366 & -0.845 & -0.029 & -0.204 & 0.908 & - & - & - \\
\hline$p$-value & 0.423 & 0.317 & 0.545 & 0.071 & 0.963 & 0.741 & 0.033 & - & - & - \\
\hline$B 2 M$ & 0.555 & 0.222 & 0.734 & -0.382 & 0.28 & -0.165 & 0.516 & 0.427 & - & - \\
\hline $\mathrm{p}$-value & 0.332 & 0.72 & 0.158 & 0.526 & 0.648 & 0.79 & 0.373 & 0.473 & - & - \\
\hline HPRT1 & 0.096 & 0.253 & 0.315 & -0.347 & 0.592 & -0.549 & 0.884 & 0.706 & 0.755 & - \\
\hline$p$-value & 0.878 & 0.681 & 0.606 & 0.567 & 0.293 & 0.338 & 0.047 & 0.183 & 0.14 & - \\
\hline BestKeeper vs. & $G A P D H$ & ACTB & RPS9 & EEF1A1 & RPS15 & RPS23 & $U X T$ & RPL4 & $B 2 M$ & HPRT1 \\
\hline coeff. of corr. [r] & 0.372 & 0.677 & 0.751 & 0.001 & 0.446 & 0.055 & 0.774 & 0.599 & 0.809 & 0.797 \\
\hline p-value & 0.538 & 0.21 & 0.144 & 0.51 & 0.451 & 0.93 & 0.124 & 0.285 & 0.097 & 0.107 \\
\hline
\end{tabular}

\section{Zanskar Horses (ZAP)}

The $\mathrm{M}$ values calculated using geNorm analysis for all the RGs in Zanskar ponies are shown in Table 3. Except, B2M and GAPDHRGs, the $\mathrm{M}$ values for all other RGs were within the acceptable limit of $<1.5$. The $\mathrm{M}$ value for all the RGs in ZAP ranged from 0.135 to 1.721. The ranking order of RGs was as follows; RPS9=RPL4 $>$ RPS23>EEF1A1 > UXT $>$ RPS15>HPRT1>ACTB> $B 2 M>G A P D H$ (Fig. 2p). The two most stable RGs with lowest $\mathrm{M}$ value were RPS9 and RPL4 (0.135) while GAPDH and B2M were the least stable RGs with $\mathrm{M}$ value of 1.712 and 1.394 respectively. Further, the $\mathrm{V}$ values for V2/3, V3/4, V4/5 and V5/6 were within the threshold limit of 0.15 . Based on geNorm analysis, the geometric mean of RPS9, RPL 4 and RPS23 RGs is likely to provide accurate normalization of gene expression data in ZAP (Fig. 2q).

In Normfinder analysis ranking of genes in high altitude ZAP from most stable to least stable was as follows: EEF1A1 (0.631), UXT (0.684), RPS9 (0.749), RPS23 (0.759), RPL4 (0.763), RPS15(1.022), ACTB (1.36), HPRT1 (1.727), B2M (1.945), GAPDH (2.795) (Fig. 2r) 
From BestKeeper algorthim, UXT gene revealed minimum SD value of 0.341 with smallest variation, followed by RPS23, RPS9, RPL4, EEF1A1, RPS15, ACTB, HPRT1, B2M and GAPDH with the SD value $0.434,0.465,0.469,0.498,0.816,1.211,1.226$, 1.914, 2.099 respectively (Table 14). The best correlation between RGs and BestKeeper was observed for $B 2 M(r=0.947)$ and ACTB $(\mathrm{r}=0.662)$ (Table 15). The high correlation values for these genes indicated their reliability as RGs.

Table 14

Analysis of parameters based quantitative cycling points (CP) for 10 candidate RGs in ZAP

\begin{tabular}{|llllllllllll|}
\hline & GAPDH & ACTB & RPS9 & EEF1A1 & RPS15 & RPS23 & UXT & RPL4 & B2M & HPRT1 \\
\hline $\mathrm{n}$ & 5 & 5 & 5 & 5 & 5 & 5 & 5 & 5 & 5 & 5 \\
\hline geo Mean [CP] & 21.69 & 17.49 & 20.99 & 19.82 & 35.03 & 17.55 & 27.02 & 18.71 & 17.65 & 24.91 \\
\hline AR Mean [CP] & 21.81 & 17.54 & 21 & 19.83 & 35.04 & 17.56 & 27.02 & 18.72 & 17.75 & 24.96 \\
\hline min [CP] & 19.69 & 16.4 & 19.84 & 18.81 & 33.61 & 16.47 & 26.17 & 17.55 & 16.06 & 22.06 \\
\hline max [CP] & 25.66 & 19.94 & 21.45 & 20.5 & 36.06 & 18.13 & 27.41 & 19.21 & 20.49 & 26.81 \\
\hline std dev [+/- CP] & 2.1 & 1.21 & 0.46 & 0.5 & 0.82 & 0.43 & 0.34 & 0.47 & 1.91 & 1.23 \\
\hline CV [\% CP] & 9.63 & 6.91 & 2.21 & 2.51 & 2.33 & 2.47 & 1.26 & 2.5 & 10.78 & 4.91 \\
\hline min [x-fold] & -4 & -2.12 & -2.22 & -2.02 & -2.67 & -2.11 & -1.8 & -2.24 & -3 & -7.21 \\
\hline max [x-fold] & 15.68 & 5.48 & 1.37 & 1.6 & 2.05 & 1.5 & 1.31 & 1.41 & 7.18 & 3.73 \\
\hline std dev [+/- x-fold] & 4.28 & 2.32 & 1.38 & 1.41 & 1.76 & 1.35 & 1.27 & 1.38 & 3.77 & 2.34 \\
\hline
\end{tabular}


Table 15

Analysis of repeated pair-wise correlation amongst genes in ZAP with BestKeeper index

\begin{tabular}{|c|c|c|c|c|c|c|c|c|c|c|}
\hline & GAPDH & ACTB & RPS9 & $E E F 1 A 1$ & RPS15 & RPS23 & $U X T$ & RPL4 & $B 2 M$ & HPRT1 \\
\hline$A C T B$ & 0.715 & - & - & - & - & - & - & - & - & - \\
\hline p-value & 0.175 & - & - & - & - & - & - & - & - & - \\
\hline RPS9 & -0.762 & -0.798 & - & - & - & - & - & - & - & - \\
\hline p-value & 0.134 & 0.106 & - & - & - & - & - & - & - & - \\
\hline EEF1A1 & -0.586 & -0.615 & 0.913 & - & - & - & - & - & - & - \\
\hline p-value & 0.299 & 0.27 & 0.031 & - & - & - & - & - & - & - \\
\hline RPS15 & -0.734 & -0.469 & 0.826 & 0.647 & - & - & - & - & - & - \\
\hline p-value & 0.158 & 0.425 & 0.085 & 0.238 & - & - & - & - & - & - \\
\hline RPS23 & -0.628 & -0.82 & 0.976 & 0.906 & 0.728 & - & - & - & - & - \\
\hline$p$-value & 0.257 & 0.089 & 0.004 & 0.034 & 0.164 & - & - & - & - & - \\
\hline$U X T$ & -0.881 & -0.922 & 0.917 & 0.8 & 0.656 & 0.876 & - & - & - & - \\
\hline p-value & 0.049 & 0.026 & 0.028 & 0.104 & 0.229 & 0.051 & - & - & - & - \\
\hline RPL4 & -0.685 & -0.777 & 0.981 & 0.966 & 0.723 & 0.979 & 0.899 & - & - & - \\
\hline p-value & 0.202 & 0.122 & 0.003 & 0.008 & 0.167 & 0.004 & 0.038 & - & - & - \\
\hline$B 2 M$ & 0.434 & 0.854 & -0.388 & -0.128 & -0.095 & -0.446 & -0.61 & -0.347 & - & - \\
\hline$p$-value & 0.466 & 0.065 & 0.519 & 0.837 & 0.879 & 0.452 & 0.275 & 0.568 & - & - \\
\hline HPRT1 & -0.503 & 0.244 & 0.046 & 0.019 & 0.415 & -0.169 & 0.073 & -0.039 & 0.433 & - \\
\hline$p$-value & 0.387 & 0.693 & 0.941 & 0.975 & 0.488 & 0.786 & 0.907 & 0.95 & 0.467 & - \\
\hline BestKeeper vs. & GAPDH & ACTB & RPS9 & EEF1A1 & RPS15 & RPS23 & $U X T$ & RPL4 & $B 2 M$ & HPRT1 \\
\hline coeff. of corr. [r] & 0.257 & 0.662 & 0.001 & 0.159 & 0.182 & 0.001 & 0.001 & 0.001 & 0.947 & 0.427 \\
\hline$p$-value & 0.676 & 0.224 & 0.894 & 0.799 & 0.77 & 0.821 & 0.533 & 0.939 & 0.014 & 0.473 \\
\hline
\end{tabular}

In RefFinder analysis, RPS9, RPL4 and UXT were overall most stable while GAPDH, B2M and HPRT1 were the least stable. Based on all the methods; geNorm Normfinder, BestKeeper and RefFinder RPS9, RPL4 and UXT were observed to be most stable RGs in ZAP.

\section{Discussion}

These days, identification of appropriate RGs is a fundamental part of gene expression studies. It has been suggested in many reports ${ }^{10,23,39}$ that there are no panel of RGs that can be used universally for normalization of gene expression data. Several studies have been highlighted the importance of proper RGs for normalization of target genes ${ }^{26,27,40}$. Although, qPCR is a sensitive and efficient technique to quantify the expression profile of genes in different experimental conditions, there are several inevitable variations including mRNA quality and expression variability, identification of appropriate normalization factors becomes obligatory for accurate quantization of target genes expression profile. It becomes more imperative in comparative expression studies between different experimental conditions. To the best of our knowledge no such study has been reported in livestock species that are adapted to high altitude regions. In our study, a total of 10 candidate RGs that 
belonged to basic cellular processes from different functional categories were evaluated for their expression stability across high altitude adapted animals like Ladakhi Cattle, Ladakhi Yak, Double hump Camel, Ladakhi Donkey, Chanthangi Goat, Zanskar Horses. The panel of stable RGs in each livestock species were; EEF1A1, RPL4, RPS23 (Ladakhi cattle); GAPDH, RPS9, ACTB (Ladakhi Yak); B2M, HPRT1, RPS23, ACTB (Ladakhi Donkey); RPS9, HPRT1 ACTB (Changthangi goat); HPRT1, $A C T B, B 2 M$ and RPS23 (Double hump camel); RPS9, RPL4, UXT (Zanskari ponies). In past, our group has reported panel of stable RGs for different experimental condition; viz., RPS9 and RPS15 were identified as stably expressed RGs in PBMCs of Sahiwal cows and Murrah buffaloes under heat stress conditions ${ }^{19}$. Similarly, both genes were also recognized as stable RGs in mammary gland of dairy cows across different stages of lactation ${ }^{20}$. Beta-2M, RPS23, RPL4 and EEF1A1 as most trustworthy RGs in heat stressed mammary explants, and mammary epithelial cells of buffaloes ${ }^{41,18}$. RPL4, EEF1A1, ACTB and GAPDH genes were found to be most stable genes in milk derived mammary epithelial cells in Sahiwal cows during different lactation stages ${ }^{42}$. Similarly, identification of stable RGs for transcriptomic studies in bulls for meat quality trait ${ }^{43}$ and muscles ${ }^{44}$ were also reported. Tanushree et al., ${ }^{45}$ identified another panel of RGs; GAPDH, RPS15 and HPRT for normalization of qPCR data in in-vitro fertilized and cloned embryos of riverine buffaloes. In the present investigation, species wise most stable RGs were identified using geNorm, Normfinder, BestKeeper and RefFinder analysis which could be quite useful in normalization of expression data in PBMC of different species adapted to high altitude environments, substantiating the importance of RGs for particular experimental conditions ${ }^{27}$. To the best of our knowledge, this is the first systematic attempt to identify panel of RGs across different species types adapted to high altitude hypoxia conditions.

\section{Conclusion}

Use of reference genes or internal control genes (ICGs) or housekeeping (HKGs) genes with constant expression level between samples in response to experimental treatment or physiological state, are now considered as effective method for normalization of transcriptional data to account for the experimental variations ${ }^{35}$. In the present study, species wise panel of RGs were identified such as ACTB, RPS15 in Ladakhi cattle; GAPDH, RPS9 in Ladakhi yak; B2M, HPRT1 in Ladakhi donkey; HPRT1, RPS9 in Changthangi goat; B2M, HPRT1 in Double hump camel and RPS9, RPL4 in Zanskar ponies. To the best of our knowledge, this is the first systematic attempt to identify panel of reference genes across different livestock species adapted to high altitude region Leh-Ladakh. The data presented here could be used as a resource to select most suitable reference for accurate normalization of transcriptional data during all future studies resembling the experimental conditions highlighted in this study.

\section{Methods}

\section{Livestock species, sampling and PBMCs isolation}

The study was conducted in line with the relevant guidelines and regulations (following the institutional animal ethics protocol of ICAR-NBAGR). All the procedures were approved by the animal ethics committee of ICAR-NBAGR. For this study, about 7-8 ml of blood was collected from 32 individuals representing 6 native livestock species that are native of Leh-Ladakh region of India and well adapted to cold arid hypoxia conditions. For sampling 6 animals each of Ladakhi cattle (LAC), Ladakhi yak (LAY) and 5 each of Ladakhi donkey (LAD), Changthangi goat (CHG), Zanskar ponies (ZAP) and Double hump camel (DHC) were randomly selected from the breeding tract of these populations. The geographical coordinates of sampling site were latitude- $34^{\circ} 9^{\prime} 9.3168^{\prime \prime} \mathrm{N}$, and longitude $77^{\circ} 34^{\prime} 37.3764^{\prime \prime} \mathrm{E}$. The blood samples were transported to the laboratory for further processing and isolation of peripheral blood mononuclear cells (PBMCs). All the sampling was done in accordance with the guidelines and regulations of Institutional Animal Ethics Committee (IAEC). The PBMCs were isolated within 2-3 hours of blood sample collection. The density gradient centrifugation procedure adopted for purification of PBMCs has been described in one of our previous publication ${ }^{25}$. The entire workflow of the experiment was showed in Fig. 3.

\section{Purification of total RNA and cDNA synthesis}


For isolation of total RNA, the purified PBMCs were suspended in $1.0 \mathrm{ml}$ Trizol reagent (Thermo Fisher Scientific, USA). After homogenization, the standard protocol based on chloroform and isopropanol extraction was followed to isolate the total RNA. The total RNA was further purified by employing silica-membrane RNeasy spin columns (Qiagen, Germany) along with on column digestion by DNase enzyme (Qiagen, Germany). The concentration and purity of extracted was measured using Nano view plus (Biohrome Spectros, USA). The integrity of each RNA sample was also confirmed by presence of $28 \mathrm{~S}$ and $18 \mathrm{~S}$ ribosomal bands on $1.5 \%$ agarose gel.

\section{cDNA synthesis and real time quantitative PCR (qPCR)}

The first strand cDNA synthesis was carried out using RevertAid First Strand cDNA Synthesis Kit (Thermo Fisher Scientific, CA, USA). First strand cDNA was synthesized using $200 \mathrm{ng}$ of purified RNA, oligo-dT (18) primer, dNTP mix, random primers, RiboLock ${ }^{T M}$ RNase inhibitor, M-MuLV reverse transcriptase supplied with RevertAid First Strand cDNA Synthesis kit (Thermo Scientific, CA, USA). The reaction for cDNA synthesis was set up using the program: $25^{\circ} \mathrm{C}$ for $5 \mathrm{~min}, 50^{\circ} \mathrm{C}$ for $60 \mathrm{~min}$, and $70^{\circ} \mathrm{C}$ for $15 \mathrm{~min}$. The cDNA sample was diluted 1:4 (v:v) with DNase/RNase-free water. Before subjecting for qPCR reactions, each of the cDNA samples was amplified using GAPDH in a semi-quantitative PCR. This step was done to ensure the quality of all the 37 first strand cDNA synthesized from PBMCs of 6 livestock species. The amplified products were checked on $2.5 \%$ agarose gel to ensure specific amplification. A total of 10 potential candidate RGs viz., GAPDH, ACTB, RPS9, RPS15, RPS23, $B 2 M, E E F 1 A 1, R P L 4, U X T$ and HPRT1 were evaluated in this study. The purpose of evaluating the stability ranking of these 10 RGs was to provide most appropriate panel of RGs in each of six livestock species of Leh-Ladakh so that any future transcriptional data could be normalized accurately. All relevant details like gene name, primer sequences, melting temperature etc. are tabulated in Table 1.

The qPCR reactions were performed in a final volume of $10 \mu \mathrm{L}$ containing $4 \mu \mathrm{L}$ diluted cDNA combined with $6 \mu \mathrm{L}$ of master mix composed of $5 \mu \mathrm{L}$ Maxima SYBR Green/ROX qPCR master mix (2X) (Fermentas Thermo, USA), $0.4 \mu \mathrm{L}$ each of $10 \mu \mathrm{M}$ forward and reverse primers, and $0.2 \mu \mathrm{L}$ DNase/RNase free water. All the reactions were performed in duplicate along with sixpoint standard curve along with non-template control with following amplification conditions; 2 min at $50^{\circ} \mathrm{C}, 10 \mathrm{~min}$ at $95^{\circ} \mathrm{C}$, 40 cycles of $15 \mathrm{~s}$ at $95^{\circ} \mathrm{C}$ (denaturation) and $1 \mathrm{~min}$ at $60^{\circ} \mathrm{C}$ (annealing+extension) in a Step one plus real time PCR instrument (ABI, California). For standard curve of each primer pair, fivefold serial dilution was made using pooled cDNA samples. The qPCR expression data for each gene was extracted in the form of crossing points and data was subjected for subsequent analysis.

\section{Identification of reference genes and statistical analysis}

In order to evaluate the expression stability of RGs in individual species, 10 candidate genes viz., GAPDH, ACTB, RPS9, RPS15, RPS23, B2M, EEF1A1, RPL4, UXT and HPRT1 from different functional categories were selected. four independent statistical approaches viz. geNorm ${ }^{35}$, Normfinder ${ }^{36}$, BestKeeper ${ }^{37}$ and RefFinder were used to identify most stable RGs.

The geNorm software measure the expression stability as $\mathrm{M}$ value which is based on overall pairwise comparison among the reference genes. The $\mathrm{M}$ value is inversely correlated to gene expression stability and ranks the RGs accordingly. In addition, pair wise variation analysis ( $V$ values) was also carried out using geNorm software to select optimal number of RGs to be used for normalization of target gene data. NormFinder algorithm determined the optimal RGs and the combination of two genes for a two-gene normalization factor with its corresponding stability value. The BestKeeper analysis is based on pairwise comparisons of raw cycle threshold (Ct), values of each gene. The result of BestKeeper analysis is displayed as standard deviation (SD) and coefficient of variance (CV). BestKeeper software calculated the descriptive statistics of every candidate gene and excludes the genes having standard deviation (SD) greater than 1 , lower the standard deviation more is the stability of genes.

The data was analysed by direct comparing the $\mathrm{Ct}$ values in geNorm and NormFinder. The relative Ct values based on comparative Ct-method were the input data for geNorm and Normfinder ${ }^{35,38}$ wherein, the average Ct value of each duplicate reaction was converted to relative quantity data [transformed using comparative Ct method as Efficiency (minimum $\mathrm{Ct}$ - sample 
${ }^{C}$ )] with the highest expression level set to 1. As input for BestKeeper analysis, the average Ct value of each duplicate reaction was used directly (without conversion to relative quantity).

\section{Declarations}

\section{Acknowledgments}

The authors duly acknowledge the Director ICAR-NBAGR for providing the research facilities for this work.

\section{Author Contribution}

M.M., M.S. and R.S.K. contributed to study conception and design. S.K.N., V.K.B. and P.V. collected the samples. M.T., P.V., G.G. and D.C. performed the experiment. M.T., P.V., N.M., V.K.B. and M.M. analysed the data. M.T., M.S. and M.M. wrote the manuscript.

\section{Funding}

The work was supported by Indian Council of Agricultural Research, New Delhi, under the National Fellow Scheme (Grant number: 27(3)/2010-HRD).

\section{Conflict of interest}

The authors declare that they have no conflicts of interest

\section{Animal ethics}

All the experimental procedure was done in accordance with the guidelines and regulations of Institutional Animal Ethics Committee (IAEC), ICAR-National bureau of animal genetic resources (ICAR-NBAGR), Karnal, Haryana, India.

\section{References}

1. Ekman, D.R., Lorenz, W.W., Przybyla, A.E., Wolfe, N.L. \& Dean, J.F. SAGE analysis of transcriptome responses in Arabidopsis roots exposed to 2, 4, 6-trinitrotoluene. Plant Physiol. 133(3), 1397-1406 (2003). https://doi.org/10.1104/pp.103.028019.

2. Eyres, I., Jaquiéry, J., Sugio, A., Duvaux, L., Gharbi, K., Zhou, J.J., Legeai, F., Nelson, M., Simon, J.C., Smadja, C.M. \& Butlin, R. Differential gene expression according to race and host plant in the pea aphid. Mol. Ecol. 25(17), 4197-4215 (2016). https://doi.org/10.1111/mec.13771.

3. Kang, L., Chen, X., Zhou, Y., Liu, B., Zheng, W., Li, R., Wang, J. \& Yu, J.The analysis of large-scale gene expression correlated to the phase changes of the migratory locust. Proc. Natl. Acad. Sci. U.S.A. 101(51), 17611-17615 (2004). https://doi.org/10.1073/pnas.0407753101.

4. Ma, Z., Guo, W., Guo, X., Wang, X. \& Kang, L. Modulation of behavioral phase changes of the migratory locust by the catecholamine metabolic pathway. Proc. Natl. Acad. Sci. U.S.A. 108(10), 3882-3887 (2011). https://doi.org/10.1073/pnas.1015098108.

5. Wang, Q., Ishikawa, T., Michiue, T., Zhu, B.L., Guan, D.W. \& Maeda, H. Stability of endogenous reference genes in postmortem human brains for normalization of quantitative real-time PCR data: comprehensive evaluation using geNorm, NormFinder, and BestKeeper. Int. J. Legal Med. 126(6), 943-952 (2012). https://doi.org/10.1007/s00414-0120774-7.

6. Hembruff, S.L., Villeneuve, D.J. \& Parissenti, A.M. The optimization of quantitative reverse transcription PCR for verification of cDNA microarray data. Anal. Biochem. 345(2), 237-249 (2005). https://doi.org/10.1016/j.ab.2005.07.014. 
7. Valasek, M.A. \& Repa, J.J. The power of real-time PCR. Am. J. Physiol. - Adv. Physiol. Educ. 29(3), 151-159 (2005). https://doi.org/10.1152/advan.00019.2005.

8. Bansal, R., Mittapelly, P., Chen, Y., Mamidala, P., Zhao, C. \& Michel, A. Quantitative RT-PCR gene evaluation and RNA interference in the brown marmorated stink bug. PloS one. 11(5), e0152730 (2016). https://doi.org/10.1371/journal.pone.0152730.

9. Sun, K., Zhang, Y., D’Alessandro, A., Nemkov, T., Song, A., Wu, H., Liu, H., Adebiyi, M., Huang, A., Wen, Y.E. \& Bogdanov, M.V. Sphingosine-1-phosphate promotes erythrocyte glycolysis and oxygen release for adaptation to high-altitude hypoxia. Nat. Commun. 7(1), 1-13 (2016). https://doi.org/10.1038/ncomms12086.

10. Bustin, S.A., Benes, V., Nolan, T. \& Pfaffl, M.W. Quantitative real-time RT-PCR-a perspective. J. Mol. Endocrinol. 34(3), 597601 (2005). https://doi.org/10.1677/jme.1.01755.

11. Yokoyama, T., Omotehara, T., Hirano, T., Kubota, N., Yanai, S., Hasegawa, C., Takada, T., Mantani, Y. \& Hoshi, N. Identification of reference genes for quantitative PCR analyses in developing mouse gonads. J. Vet. Med. Sci. 80(10), 1534-1539 (2018). https://doi.org/10.1292/jvms.18-0417.

12. Everaert, B.R., Boulet, G.A., Timmermans, J.P. \& Vrints, C.J. Importance of suitable reference gene selection for quantitative real-time PCR: special reference to mouse myocardial infarction studies. PloS one. 6(8), e23793 (2011). https://doi.org/10.1371/journal.pone.0023793.

13. Acimovic, J., Golicnik, M., Perse, M., Majdic, G., Fink, M. \& Rozman, D. Determination of reference genes for circadian studies in different tissues and mouse strains. BMC Mol. Biol. 11(1):1-1 (2010). https://doi.org/10.1186/1471-2199-1160 .

14. Oturai, D.B., Søndergaard, H.B., Börnsen, L., Sellebjerg, F. \& Romme Christensen, J. Identification of suitable reference genes for peripheral blood mononuclear cell subset studies in multiple sclerosis. Scand. J. Immunol. 83(1), 72-80 (2016). https://doi.org/10.1111/sji.12391.

15. Hong, S.Y., Seo, P.J., Yang, M.S., Xiang, F. \& Park, C.M. Exploring valid reference genes for gene expression studies in Brachypodium distachyon by real-time PCR. BMC Plant Biol. 8(1), 1-11 (2008). https://doi.org/10.1186/1471-2229-8-112.

16. Czechowski, T., Stitt, M., Altmann, T., Udvardi, M.K. \& Scheible, W.R. Genome-wide identification and testing of superior reference genes for transcript normalization in Arabidopsis. Plant Physiol. 139(1), 5-17 (2005). https://doi.org/10.1104/pp.105.063743.

17. Kaur, R., Sodhi, M., Sharma, A., Sharma, V.L., Verma, P., Swami, S.K., Kumari, P. \& Mukesh, M. Selection of suitable reference genes for normalization of quantitative RT-PCR (RT-qPCR) expression data across twelve tissues of riverine buffaloes (Bubalus bubalis). PloS one. 13(3), e0191558 (2018). https://doi.org/10.1371/journal.pone.0191558.

18. Kapila, N., Kishore, A., Sodhi, M., Sharma, A., Kumar, P., Mohanty, A.K., Jerath, T. \& Mukesh, M. Identification of appropriate reference genes for qRT-PCR analysis of heat-stressed mammary epithelial cells in riverine buffaloes (Bubalus bubalis). ISRN Biotechnol. 2013, 1-9 (2013) https://doi.org/10.5402/2013/735053.

19. Kishore, A., Sodhi, M., Kumari, P., Mohanty, A.K., Sadana, D.K., Kapila, N., Khate, K., Shandilya, U., Kataria, R.S. \& Mukesh, M. Peripheral blood mononuclear cells: a potential cellular system to understand differential heat shock response across native cattle (Bos indicus), exotic cattle (Bos taurus), and riverine buffaloes (Bubalus bubalis) of India. Cell Stress and Chaperones 19(5), 613-621 (2014). https://doi.org/10.1007/s12192-013-0486-z.

20. Bionaz, M. \& Loor, J.J. Identification of reference genes for quantitative real-time PCR in the bovine mammary gland during the lactation cycle. Physiol. Genomics. 29(3), 312-319 (2007).

https://doi.org/10.1152/physiolgenomics.00223.2006.

21. Li, Q., Domig, K.J., Ettle, T., Windisch, W., Mair, C. \& Schedle, K. Evaluation of potential reference genes for relative quantification by RT-qPCR in different porcine tissues derived from feeding studies. Int. J. Mol. Sci. 12(3), 1727-1734 (2011). https://doi.org/10.3390/ijms12031727.

22. Guénin, S., Mauriat, M., Pelloux, J., Van Wuytswinkel, O., Bellini, C. \& Gutierrez, L. Normalization of qRT-PCR data: the necessity of adopting a systematic, experimental conditions-specific, validation of references. J. Exp. Bot. 60(2), 487-493

Page 25/30 
(2009). https://doi.org/10.1093/jxb/ern305.

23. Dheda, K., Huggett, J.F., Chang, J.S., Kim, L.U., Bustin, S.A., Johnson, M.A., Rook, G.A.W. \& Zumla, A. The implications of using an inappropriate reference gene for real-time reverse transcription PCR data normalization. Anal. Biochem. 344(1), 141-143 (2005). https://doi.org/10.1016/j.ab.2005.05.022.

24. Verma, P., Sharma, A., Sodhi, M., Thakur, K., Kataria, R.S., Niranjan, S.K., Bharti, V.K., Kumar, P., Giri, A., Kalia, S. \& Mukesh, M. Transcriptome analysis of circulating PBMCs to understand mechanism of high altitude adaptation in native cattle of Ladakh region. Sci. Rep. 8(1), 1-15 (2018). https://doi.org/10.1038/s41598-018-25736-7.

25. Verma, P., Sharma, A., Sodhi, M., Thakur, K., Bharti, V.K., Kumar, P., Giri, A., Kalia, S., Swami, S.K. \& Mukesh, M. Overexpression of genes associated with hypoxia in cattle adapted to Trans Himalayan region of Ladakh. Cell Biol. Int. 42(9), 1141-1148 (2018). https://doi.org/10.1002/cbin.10981.

26. De Ketelaere, A., Goossens, K., Peelman, L. \& Burvenich, C. Validation of internal control genes for gene expression analysis in bovine polymorphonuclear leukocytes. J. Dairy Sci. 89(10), 4066-4069 (2006). https://doi.org/10.3168/jds.S0022-0302(06)72450-X.

27. Jiang, M., Lee, J.N., Bionaz, M., Deng, X.Y. \& Wang, Y. Evaluation of suitable internal control genes for RT-qPCR in yak mammary tissue during the lactation cycle. PLoS One. 11(1), e0147705 (2016). https://doi.org/10.1371/journal.pone.0147705.

28. Nygard, A.B., Jørgensen, C.B., Cirera, S. \& Fredholm, M. Selection of reference genes for gene expression studies in pig tissues using SYBR green qPCR. BMC Mol. Biol. 8(1), 1-6 (2007). https://doi.org/10.1186/1471-2199-8-67.

29. Zhang, Y., Zhang, X.D., Liu, X., Li, Y.S., Ding, J.P., Zhang, X.R. \& Zhang, Y.H. Reference gene screening for analyzing gene expression across goat tissue. Asian-Australasian J. Anim. Sci. 26(12). 1665-1671 (2013).

https://doi.org/10.5713/ajas.2013.13199.

30. Sahu, A.R., Wani, S.A., Saxena, S., Rajak, K.K., Chaudhary, D., Sahoo, A.P., Khanduri, A., Pandey, A., Mondal, P., Malla, W.A. \& Khan, R.I.N. Selection and validation of suitable reference genes for qPCR gene expression analysis in goats and sheep under Peste des petits ruminants virus (PPRV), lineage IV infection. Sci. Rep. 8(1), 1-11 (2018). https://doi.org/10.1038/s41598-018-34236-7.

31. Vorachek, W.R., Bobe, G. \& Hall, J.A. Reference gene selection for quantitative PCR studies in sheep neutrophils. Int. J. Mol. Sci. 14(6), 11484-11495 (2013). https://doi.org/10.3390/ijms140611484.

32. Cappelli, K., Felicetti, M., Capomaccio, S., Spinsanti, G., Silvestrelli, M. \& Supplizi, A.V. Exercise induced stress in horses: selection of the most stable reference genes for quantitative RT-PCR normalization. BMC Mol. Biol. 9(1), 1-8 (2008). https://doi.org/10.1186/1471-2199-9-49.

33. Nakamura, A.M., Chahad-Ehlers, S., Lima, A.L.A., Taniguti, C.H., Sobrinho Jr, I., Torres, F.R. \& de Brito, R.A. Reference genes for accessing differential expression among developmental stages and analysis of differential expression of OBP genes in Anastrepha obliqua. Sci. Rep. 6(1), 1-10 (2016). https://doi.org/10.1038/srep17480.

34. Robledo Sánchez, D., Hernández Urcera, J., Cal, R., Gómez Pardo, M.B., Sánchez Piñón, L.E., Martínez Portela, P. \& Viñas Díaz, A.M. Analysis of qPCR reference gene stability determination methods and a practical approach for efficiency calculation on a turbot (Scophthalmus maximus) gonad dataset. BMC Genomics. 15(1), 1-15 (2014). https://doi.org/10.1186/1471-2164-15-648.

35. Vandesompele, J., De Preter, K., Pattyn, F., Poppe, B., Van Roy, N., De Paepe, A. \& Speleman, F. Accurate normalization of real-time quantitative RT-PCR data by geometric averaging of multiple internal control genes. Genome Biol. 3(7), 1-12 (2002).

36. Andersen, C.L., Jens L.J., and Torben F.Ø. "Normalization of real-time quantitative reverse transcription-PCR data: a model-based variance estimation approach to identify genes suited for normalization, applied to bladder and colon cancer data sets." Cancer research 64.15 5245-5250 (2004).

37. Pfaffl, M.W., Tichopad, A., Prgomet, C., \& Neuvians, T.P. Determination of stable housekeeping genes, differentially regulated target genes and sample integrity: BestKeeper-Excel-based tool using pair-wise correlations. Biotechnology

Page 26/30 
letters, 26(6), 509-515(2004).

38. Livak, K.J. \& Schmittgen, T.D. Analysis of relative gene expression data using real-time quantitative PCR and the 2- $\triangle \triangle C T$ method. Methods. 25(4), 402-408 (2001). https://doi.org/10.1006/meth.2001.1262.

39. Huggett, J., Dheda, K., Bustin, S. \& Zumla, A. Real-time RT-PCR normalisation; strategies and considerations. Genes Immun. 6(4), 279-284 (2005). https://doi.org/10.1038/sj.gene.6364190.

40. Castigliego, L., Armani, A., Li, X., Grifoni, G., Gianfaldoni, D. \& Guidi, A. Selecting reference genes in the white blood cells of buffalos treated with recombinant growth hormone. Anal. Biochem. 403(1-2), 120-122 (2010).

https://doi.org/10.1016/j.ab.2010.04.001.

41. Sodhi, M., Kishore, A., Khate, K., Kapila, N., Mishra, B.P., Kataria, R.S., Mohanty, A.K., Varshney, N. \& Mukesh, M. Evaluating suitable internal control genes for transcriptional studies in heat-stressed mammary explants of buffaloes. J. Anim. Breed. Genet. 130(2), 106-117 (2013). https://doi.org/10.1111/j.1439-0388.2012.01004.x.

42. Jatav, P., Sodhi, M., Sharma, A., Mann, S., Kishore, A., Shandilya, U.K., Mohanty, A.K., Kataria, R.S., Yadav, P., Verma, P. \& Kumar, S. Identification of internal control genes in milk-derived mammary epithelial cells during lactation cycle of I ndian zebu cow. Anim. Sci. J. 87(3), 344-353 (2016). https://doi.org/10.1111/asj.12384.

43. Lisowski, P., Pierzchała, M., Gościk, J., Pareek, C.S. \& Zwierzchowski, L. Evaluation of reference genes for studies of gene expression in the bovine liver, kidney, pituitary, and thyroid. J. Appl. Genet. 49(4), 367-372 (2008). https://doi.org/10.1007/BF03195635.

44. Vibha, K., Ali, A.S., Sushma, P., Manishi, M., Tantia, M.S. \& Dahiya, S.S. Evaluation of internal control genes for gene expression studies in skeletal muscle of riverine buffaloes. Indian J Ani Sci. 83(9), 942-947 (2013).

45. Sood, T.J., Lagah, S.V., Sharma, A., Singla, S.K., Mukesh, M., Chauhan, M.S., Manik, R. \& Palta, P. Selection of suitable internal control genes for accurate normalization of real-time quantitative PCR data of buffalo (Bubalus bubalis) blastocysts produced by SCNT and IVF. Cell. Reprogram.19(5), 302-310 (2017). https://doi.org/10.1089/cell.2017.0010.

\section{Figures}



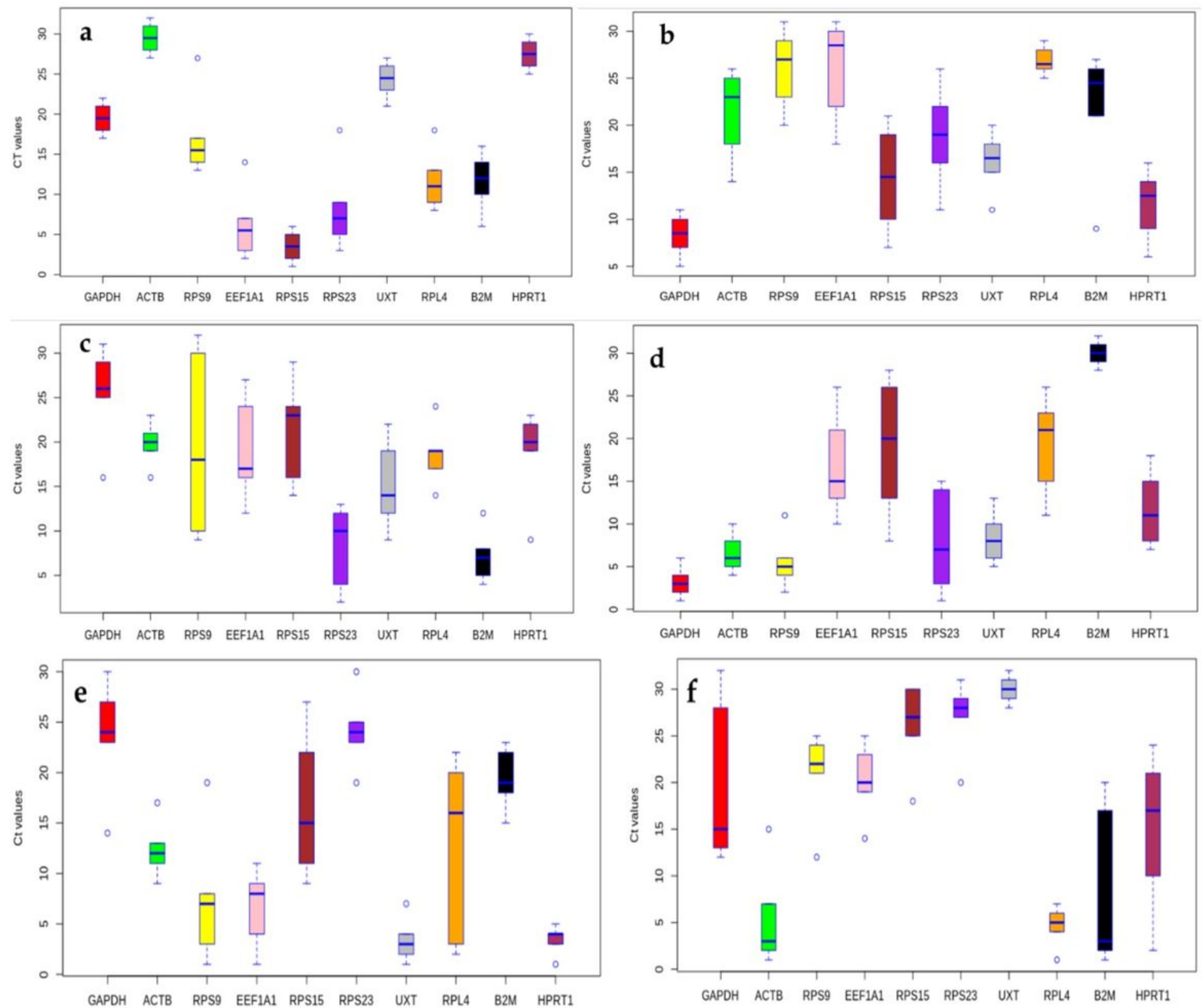

Figure 1

Expression levels of individual candidate RGs in LAC (a), LAY (b), LAD (c), CHG (d), DHC (e) and ZAP (f). The data is presented as quantification cycle $(\mathrm{Cq})$ values of each gene in the box-whisker diagram. The median is shown as a line across the box while whiskers indicate maximum and minimum values 

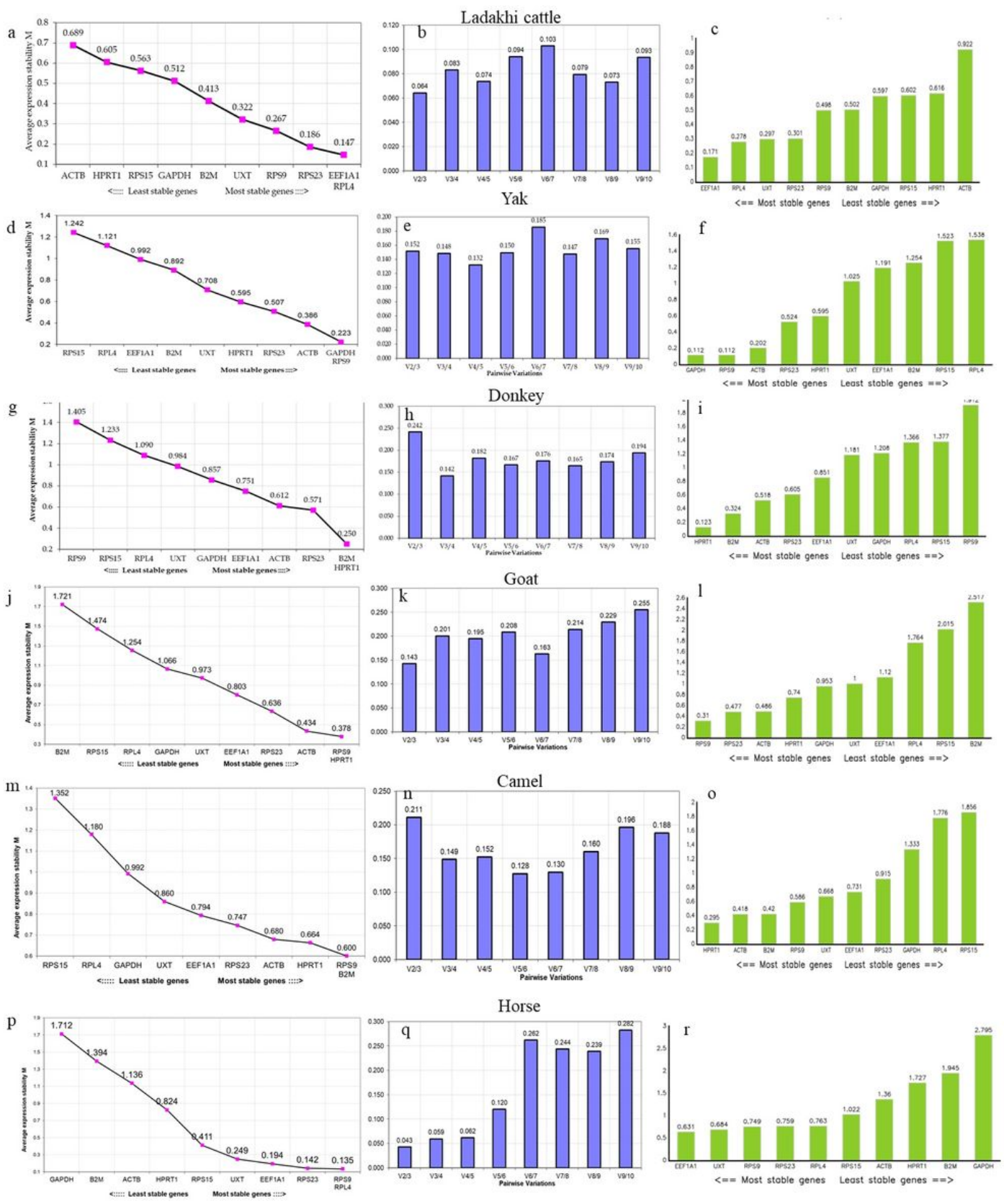

Figure 2

GeNorm analysis for ranking of genes based on average expression stability measure (M value), Pair-wise variation ( $V n / V n+$ 1) between the normalization factors NFn and NFn +1 to determine the optimal number of reference genes and Normfinder Aanlysis in LAC ( $a, b$ \& c), LAY (d, e \& f), LAD (, h \& i), CHG (j, k \& l), DHC (m, n \& o) and ZAP (p, q \& r respectively). 


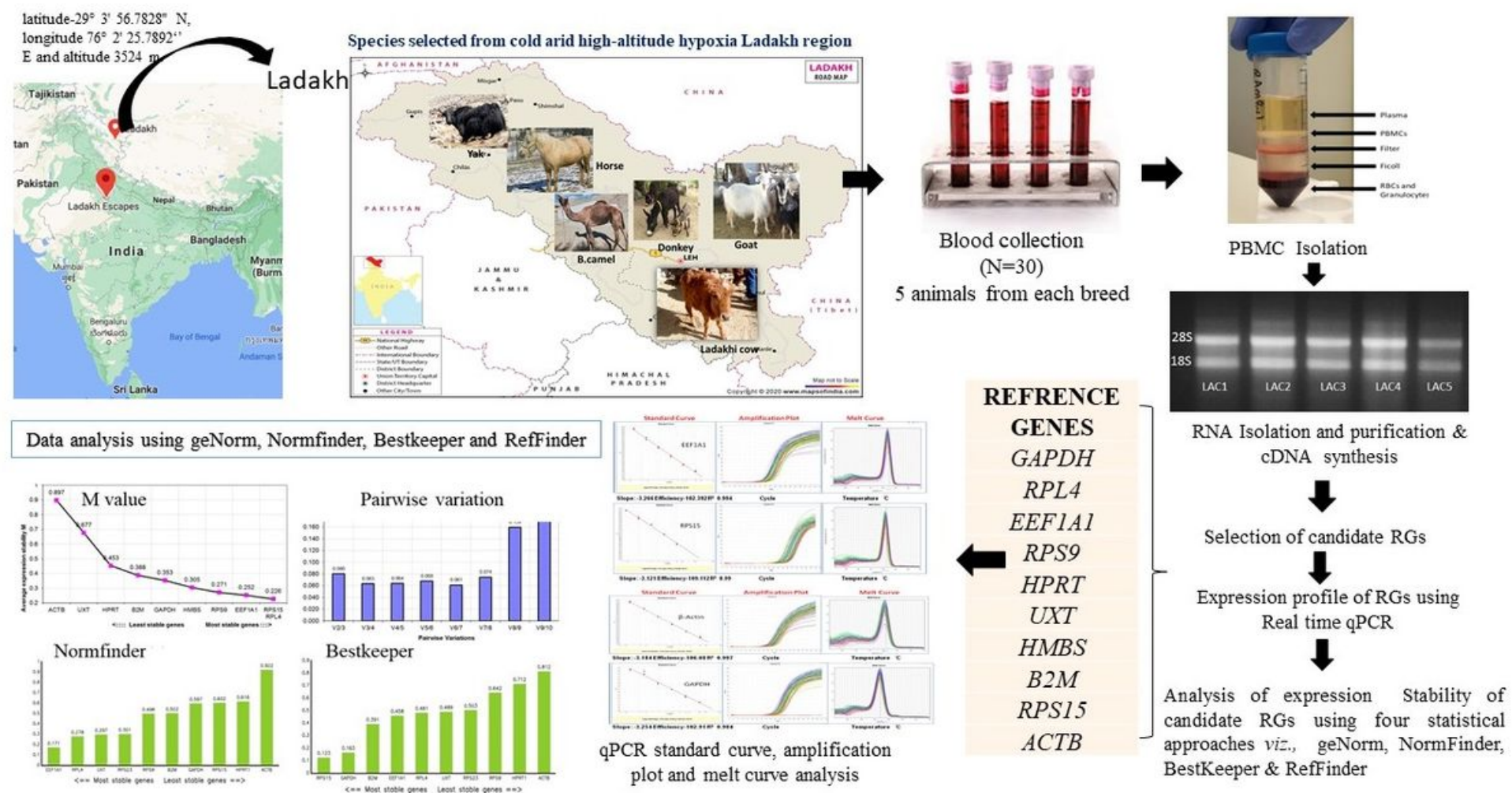

\section{Figure 3}

The entire workflow of the qPCR experiment conducted in PBMCs of different species adapted to cold arid hypoxia environment

\section{Supplementary Files}

This is a list of supplementary files associated with this preprint. Click to download.

- supplementryfileAcrossthespecies23.11.21.pdf 